\title{
Oak genome reveals facets of long lifespan
}

\author{
Christophe Plomion ${ }^{1,24 \star}$, Jean-Marc Aury ${ }^{2024}$, Joëlle Amselem ${ }^{3,24}$, Thibault Leroy ${ }^{1}$, \\ Florent Murat ${ }^{4}$, Sébastien Duplessis ${ }^{5}{ }^{5}$, Sébastien Faye ${ }^{2}$, Nicolas Francillonne ${ }^{3}$, Karine Labadie ${ }^{2}$, \\ Grégoire Le Provost ${ }^{1}$, Isabelle Lesur ${ }^{1,6}$, Jérôme Bartholomé ${ }^{1}{ }^{1}$, Patricia Faivre-Rampant ${ }^{7}$, \\ Annegret Kohler ${ }^{5}$, Jean-Charles Leplé ${ }^{8}$, Nathalie Chantret ${ }^{9}$, Jun Chen ${ }^{10}$, Anne Diévart ${ }^{11,12}$, \\ Tina Alaeitabar ${ }^{3}$, Valérie Barbe ${ }^{2}$, Caroline Belser ${ }^{(1)}{ }^{2}$, Hélène Bergès ${ }^{13}$, Catherine Bodénès ${ }^{1}$, \\ Marie-Béatrice Bogeat-Triboulot ${ }^{14}$, Marie-Lara Bouffaud ${ }^{15}$, Benjamin Brachi ${ }^{\circledR 1}$, Emilie Chancerel ${ }^{1}$, \\ David Cohen ${ }^{14}$, Arnaud Couloux ${ }^{2}$, Corinne Da Silva², Carole Dossat ${ }^{2}$, François Ehrenmann', \\ Christine Gaspin ${ }^{16}$, Jacqueline Grima-Pettenati ${ }^{17}$, Erwan Guichoux ${ }^{1}$, Arnaud Hecker5, \\ Sylvie Herrmann ${ }^{18}$, Philippe Hugueney ${ }^{19}$, Irène Hummel ${ }^{14}$, Christophe Klopp ${ }^{16}$, Céline Lalanne ${ }^{1}$, \\ Martin Lascoux ${ }^{10}$, Eric Lasserre ${ }^{20}$, Arnaud Lemainque ${ }^{2}$, Marie-Laure Desprez-Loustau', \\ Isabelle Luyten ${ }^{3}$, Mohammed-Amin Madoui' ${ }^{2}$, Sophie Mangenot ${ }^{2}$, Clémence Marchal ${ }^{5}$, \\ Florian Maumus ${ }^{3}$, Jonathan Mercier ${ }^{2}$, Célia Michotey ${ }^{3}$, Olivier Panaud ${ }^{20}$, Nathalie Picault ${ }^{20}$, \\ Nicolas Rouhier ${ }^{5}$, Olivier Rué ${ }^{16}$, Camille Rustenholz ${ }^{19}$, Franck Salin ${ }^{1}$, Marçal Soler ${ }^{17,21}$, Mika Tarkka ${ }^{15}$, \\ Amandine Velt ${ }^{19}$, Amy E. Zanne ${ }^{22}$, Francis Martin ${ }^{15}{ }^{5}$, Patrick Wincker ${ }^{23}$, Hadi Quesneville ${ }^{3}$, \\ Antoine Kremer ${ }^{1}$ and Jérôme Salse ${ }^{4}$
}

\begin{abstract}
Oaks are an important part of our natural and cultural heritage. Not only are they ubiquitous in our most common landscapes ${ }^{1}$ but they have also supplied human societies with invaluable services, including food and shelter, since prehistoric times ${ }^{2}$. With 450 species spread throughout Asia, Europe and America $^{3}$, oaks constitute a critical global renewable resource. The longevity of oaks (several hundred years) probably underlies their emblematic cultural and historical importance. Such long-lived sessile organisms must persist in the face of a wide range of abiotic and biotic threats over their lifespans. We investigated the genomic features associated with such a long lifespan by sequencing, assembling and annotating the oak genome. We then used the growing number of whole-genome sequences for plants (including tree and herbaceous species) to investigate the parallel evolution of genomic characteristics potentially underpinning tree longevity. A further consequence of the long lifespan of trees is their accumulation of somatic mutations during mitotic divisions of stem cells present in the shoot apical meristems. Empirical ${ }^{4}$
\end{abstract}

and modelling ${ }^{5}$ approaches have shown that intra-organismal genetic heterogeneity can be selected for ${ }^{6}$ and provides direct fitness benefits in the arms race with short-lived pests and pathogens through a patchwork of intra-organismal phenotypes $^{7}$. However, there is no clear proof that large-statured trees consist of a genetic mosaic of clonally distinct cell lineages within and between branches. Through this case study of oak, we demonstrate the accumulation and transmission of somatic mutations and the expansion of disease-resistance gene families in trees.

We sequenced the highly heterozygous genome of pedunculate oak (Quercus robur L.; Supplementary Notes 1 and 2) using a combination of long and short sequence reads (Supplementary Table 1). We generated a highly contiguous haploid genome sequence of a heterozygous tree comprising 1,409 nuclear scaffolds, with an N50 of $1.35 \mathrm{Mb}$ (Supplementary Note 2.2, Supplementary Table 2, Supplementary Fig. 1). A comparison with existing tree genomes is shown in Supplementary Table 3. In total, 871 scaffolds, covering $96 \%(716.6 \mathrm{Mb})$ of the estimated

'BIOGECO, INRA, Université de Bordeaux, Cestas, France. ${ }^{2}$ Commissariat à I'Energie Atomique (CEA), Genoscope, Institut de Biologie François-Jacob, Evry, France. ${ }^{3}$ URGI, INRA, Université Paris-Saclay, Versailles, France. ${ }^{4}$ GDEC, INRA-UCA, Clermont-Ferrand, France. ${ }^{5}$ IAM, INRA, Université de Lorraine, Champenoux, France. ${ }^{6} \mathrm{Helix}$ Venture, Mérignac, France. ${ }^{7}$ INRA, US 1279 EPGV, Université Paris-Saclay, Evry, France. ${ }^{8}$ BIOFORA, INRA, Orléans, France. ${ }^{9}$ AGAP, Université de Montpellier, CIRAD, INRA, Montpellier SupAgro, Montpellier, France. ${ }^{10}$ Department of Ecology and Genetics, Evolutionary Biology Centre, Science for Life Laboratory, Uppsala University, Uppsala, Sweden. ${ }^{11} \mathrm{CIRAD}$, UMR AGAP, Montpellier, France. ${ }^{12}$ Université de Montpellier, CIRAD, INRA, Montpellier SupAgro, Montpellier, France. ${ }^{13} \mathrm{CNRGV}$, INRA, Castanet, France. ${ }^{14}$ UMR Silva, INRA, Université de Lorraine, AgroPariTech, Nancy, France. ${ }^{15}$ Department of Soil Ecology, UFZ-Helmholtz Centre for Environmental Research, Halle/Saale, Germany. ${ }^{16}$ Plateforme bioinformatique Toulouse Midi-Pyrénées, INRA, Auzeville Castanet-Tolosan, France. ${ }^{17}$ Université de Toulouse, CNRS, UMR 5546, LRSV, Castanet-Tolosan, France. ${ }^{18}$ German Centre for Integrative Research (iDiv), Halle-Jena-Leipzig, Leipzig, Germany. ${ }^{19} \mathrm{SVQV}$, Université de Strasbourg, INRA, Colmar, France. ${ }^{20}$ Université de Perpignan, UMR 5096, Perpignan, France. ${ }^{21}$ Laboratori del Suro, University of Girona, Girona, Spain. ${ }^{22}$ Department of Biological Sciences, George Washington University, Washington, DC, USA. ${ }^{23}$ Génomique Métabolique, Genoscope, Institut de Biologie François-Jacob, Commissariat à l'Energie Atomique (CEA), CNRS, Université d'Evry, Université Paris-Saclay, Evry, France. ${ }^{24}$ These authors contributed equally: Christophe Plomion, Jean-Marc Aury, Joëlle Amselem. *e-mail: christophe.plomion@inra.fr 


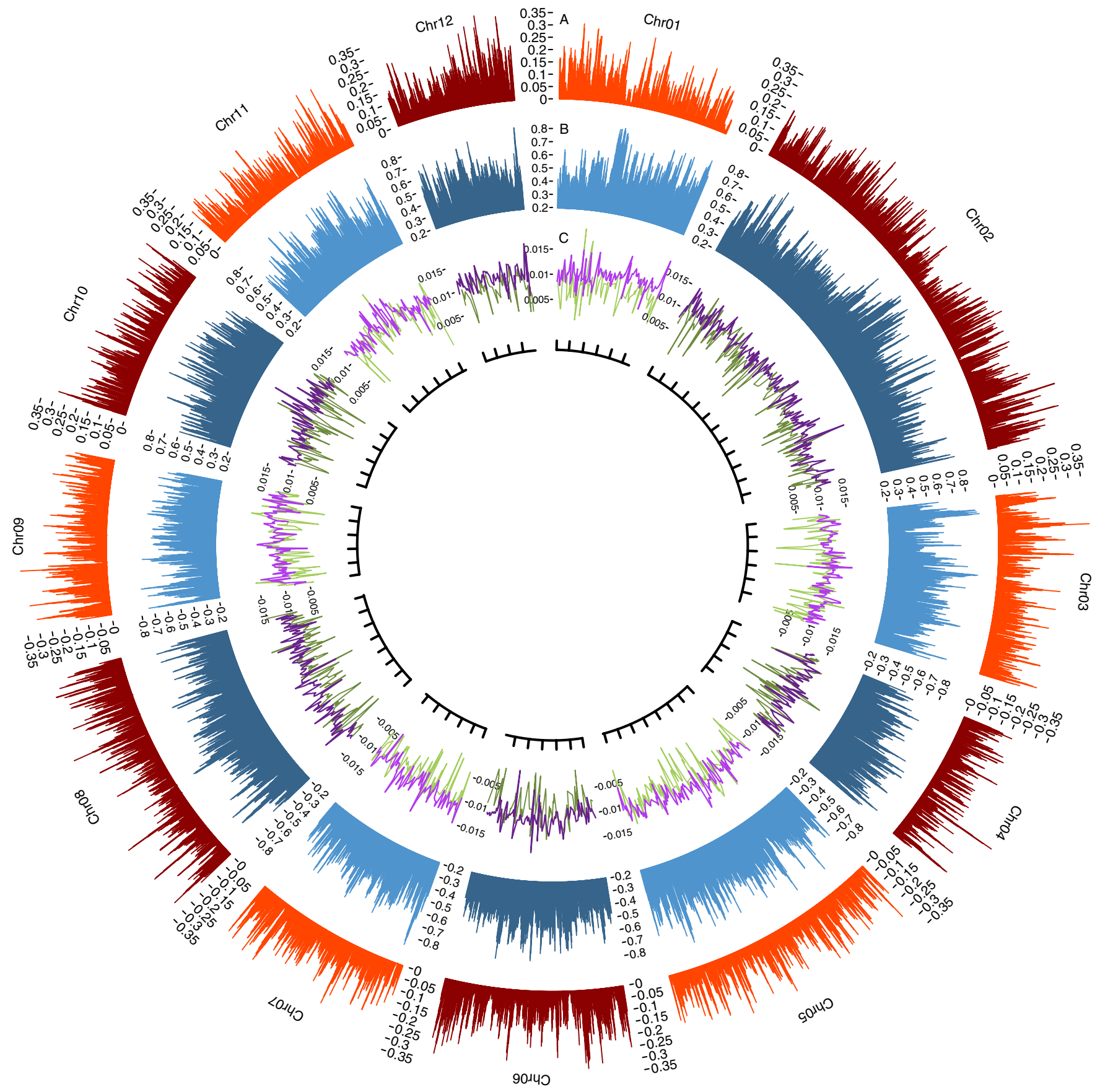

Fig. 1 Genomic landscape of the 12 assembled oak chromosomes. Gene (A) and TE (B) density, percentage heterozygosity (purple in C) and genetic diversity (green in C). These four metrics are calculated in 1-Mb sliding windows, moved in 250-kb steps. A ruler is drawn on each chromosome, with tick marks every $10 \mathrm{Mb}$.

physical size of the oak genome and containing $90 \%$ of the 25,808 predicted protein-coding genes (Supplementary Data Set 1, Supplementary Note 3.3), were anchored to the 12 oak chromosomes. To this end, we used the existing high-density oak genebased linkage map ${ }^{8}$ combined with a synteny-driven approach using Prunus persica as a pivotal genome. Non-anchored scaffolds harbouring genes syntenic to peach were placed on the pseudomolecules based on the local microsynteny identified between oak and peach (Fig. 1, Supplementary Note 2.3, Supplementary Fig. 2, Supplementary Data Set 2). Overall, $52 \%$ of the genome was found to consist of diverse transposable elements (TEs), which were dominated by class I retrotransposons (70\%) (Supplementary Table 4, Supplementary Fig. 3, Supplementary Notes 3.1 and 3.4). Genome-wide genetic diversity, as assessed by an analysis of single-nucleotide polymorphisms (SNPs) at the individual level (heterozygosity rate) and using a population of 20 genotypes $(\pi)$, amounted to $\sim 1 \%$, with significant variation within and between chromosomes (Fig. 1, Supplementary Fig. 4). Nucleotide diversity in protein-coding genes was 0.011 for fourfold degenerate sites and 0.005 for non-degenerate sites, with a non-synonymous-tosynonymous nucleotide diversity ratio $\left(\pi_{0} / \pi_{4}\right)$ of 0.44 . A comparison of these values with those obtained in a recent survey of plant 

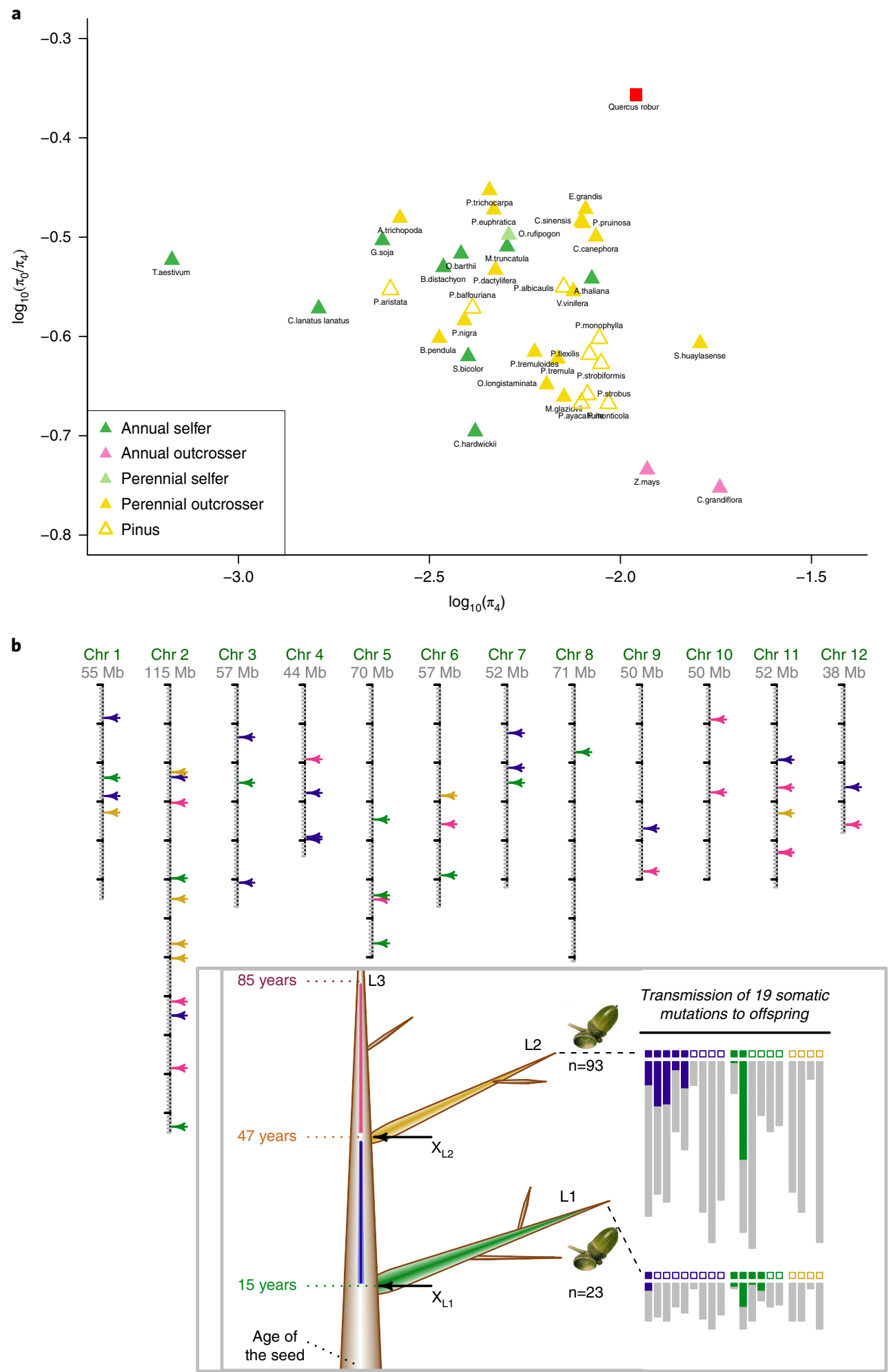

Fig. 2 | Genetic diversity and somatic mutations. a, Distribution of $\pi_{0} / \pi_{4}$ plotted against $\pi_{4}$ among plants (modified from a previous publication ${ }^{9}$ ), including oak (red square). Species names are according to ${ }^{9}$. b. Genomic location of somatic mutations along the 12 chromosomes of a 100 -year-old oak tree. Mutations are represented as coloured arrows according to where they took place during tree growth (see inset). Location and age (left of the trunk) of the three levels (L1, L2 and L3) sampled for somatic mutation detection in the reference pedunculate oak genotype 3P. L1, L2 and L3 represent the end of selected branches; $X_{L 1}$ and $X_{12}$ represent L1-branch and L2-branch initiation sites, respectively. For each branch, the recovery or non-recovery of mutations in acorns is indicated by filled and open squares, respectively. The numbers of copies of the alternative (coloured) and reference (grey) alleles are shown below each square.

and animal species ${ }^{9}$ indicated that oak was remarkable in terms of both its high nucleotide diversity $\left(\pi_{4}\right)$ and the high rate at which it accumulates deleterious mutations (Fig. 2a, Supplementary Note
4.1). Indeed, the value for oak shows the largest deviation from the regression line, with the largest residual (0.25) compared with the other 37 plant species (ranging from -0.13 to 0.12 ). 
In addition to the spontaneous meiotic mutations in each generation, long-lived plants are expected to accumulate somatic mutations throughout their lifetime. These mutations occur during the mitotic divisions of stem cells in the shoot apical meristems ${ }^{4}$. In trees, unlike animals, these mutations can be passed from the soma to the reproductive tissue and on to the offspring. Somatic mutations may therefore increase genetic diversity in long-lived trees such as oaks. Oaks have weak apical control (that is, an inability to control the flushing and growth of lateral buds from the previous year ${ }^{10}$ ), resulting in a multi-stemmed morphology. As such, oaks constitute a particularly appropriate model for studies of the somatic generation of diversity. We sampled buds at the extremities of branches initiated at the ages 15, 47 and 85 years on the reference tree sequenced in this study (Fig. 2b, Supplementary Fig. 5). Using a frequency-dependent method for detecting somatic point mutations in genomic $\mathrm{DNA}^{11}$, we identified 46 reliable somatic mutations (Supplementary Note 4.2, Supplementary Table 5) most of which (44) were located on scaffolds anchored to the 12 chromosomes (Fig. 2b). Compared with a recent report that also used the pedunculate oak as a model system ${ }^{12}$, we detected 2.7 times more somatic mutations on a tree that was 3 times younger. This difference is probably due to our superior ability to detect somatic mutations on a higher fraction of the genome (owing to the quality of our genome assembly) and smaller changes in allele frequency by applying a frequency-explicit method. This method was developed for cancer research and, in our case, accounts for the mosaic of mutated and non-mutated stem cells in shoot apical meristems. Given that most somatic mutations have a low allele frequency $(1 / 2 \mathrm{~N}$ stem cells) during growth ${ }^{13}$, most somatic mutations are expected to remain at frequencies too low to be unambiguously detected. Thus, while this work provides clear evidence that somatic mutations exist in trees, it still remains particularly challenging to determine the actual rate of somatic mutations. Consequently, we consider that the number of somatic mutations identified in the studied genotype reported here is only the tip of the iceberg of the total number of somatic mutations. A previous study ${ }^{12}$ formulated an interesting working hypothesis whereby stem cell mutagenesis protects shoot apical meristems against ultraviolet damage. This hypothesis was based on the discrepancy between theoretical expectations and the low number of empirically identified somatic mutations. However, considering the detection bias for low allele frequency variants, the hypothesis remains unsupported even with the best genomic data available to date. We then investigated the transmission of mutations to the offspring by evaluating a subset of 19 somatic mutations (Supplementary Table 6) in 116 acorns collected from the extremities of lateral branches (Fig. 2b). Despite the limited number of seeds collected, we recovered $47 \%$ (9/19) of the somatic mutations in the embryonic tissues of the acorns, confirming intergenerational transmission (Fig. 2b). Our work demonstrates that somatic mutations exist in oak and are passed onto the next generation. However, our results do not allow conclusions to be drawn on the contribution of somatic mutations to the high genetic diversity level and large-scale evolution of oaks.

We searched for genomic features specific to oak that might contribute to its longevity by first reconstructing its paleohistory within the rosid clade. We compared the ancestral eudicot karyotype $\left(\mathrm{AEK}^{14}\right)$ reconstructed from a comparison of the Vitales $\left(\right.$ grape $\left.^{15}\right)$, Rosales $\left(\right.$ peach $^{16}$ ) and Malvales ( $\operatorname{cocoa}^{17}$ ) major subfamilies to reveal that oak experienced 5 fissions and 14 fusions from 21 AEK $^{18}$ chromosomes to reach the modern 12 chromosomes (Fig. 3a). The synonymous substitution rate $\left(K_{\mathrm{s}}\right)$ of paralogues (Fig. $\left.3 \mathrm{~b}\right)$ indicated that oak did not experience lineage-specific whole-genome duplication in addition to the ancestral triplication shared among the eudicots $\left(\gamma^{19}\right)$. We also found that oak experienced a recent burst of local gene duplications (accounting for $35.6 \%$ of the oak gene repertoire) after the oak-peach lineage diverged (Fig. 3b). The eucalyptus genome is the only other plant genome shown to date to display such high levels of tandem duplication ${ }^{20}(34 \%)$, contrasting strongly with the other four genomes investigated ( $<25 \%$ tandem duplicates). We next validated that recent tandemly duplicated genes (TDGs) were true duplicates rather than different alleles or duplication artefacts generated during haplome construction (that is, during the scaffolding or merging steps of our hierarchical assembly pipeline). To this end, we applied two verification procedures based on a comparison of polymorphisms of allelic gene pairs (Supplementary Fig. 22) and a sequence coverage analysis (Supplementary Fig. 23).

A comparison of gene families $(36,844$ orthogroups, including 435,095 genes from 16 plant species (Supplementary Table 7)) provided further clues to the functional significance of tandem duplications. Of the 524 orthogroups found to have undergone expansion in oak relative to the other 15 species (Supplementary Data Set 3), 73\% of the genes of concerned were tandem duplicates (Supplementary Data Set 4). Such a tight relationship between TDGs and lineagespecific selection is not a novel observation ${ }^{21}$, and it seems to be particularly common for disease-resistance (R) genes ${ }^{22}$. However, the higher frequency of such relationships in long-lived plants, such as oak and eucalyptus, suggests that there may be a convergent mechanism in trees towards an expansion of these families of genes in long-lived species.

The orthogroups expanded in oaks are clearly enriched in Gene Ontology (GO) terms relating to biotic interactions. They included $95 \%$ of the 1,091 nucleotide-binding site leucine-rich repeat (NBLRR)-related protein genes and 55\% of the 1,247 receptor-like kinase (RLK)-encoding genes (Supplementary Data Sets 5 and 6, Supplementary Table 8, Supplementary Notes 3.5.6 and 3.5.7). We detected a particularly strong expansion of two major clades of toll interleukin receptor (TIR)-NB-LRRs in orthogroup 1 (shaded areas in Fig. 4a and Supplementary Fig. 6). In addition, three of the nine orthogroups displaying the strongest expansions (Fig. 3d, Supplementary Data Set 3) corresponded to intracellular receptors (NB-LRRs for orthogroups 1, 2 and 8) and four corresponded to cell surface receptors of the innate immune response (RLKs for orthogroups 3, 6 and 9, and LRR-receptor-like protein (RLP) for orthogroup 5). The entire complement of NB-LRR and RLK genes accounted for $9 \%$ of all oak genes, a proportion that is approximately twice that reported for other plants ${ }^{23,24}$. Moreover, $75 \%$ and $65 \%$ of the NB-LRR and RLK expansions, respectively, can be accounted for by tandem duplications. The distribution of the LRR-RLK genes between the established subgroups based on an analysis of 31 angiosperm ${ }^{25}$ also revealed remarkable expansions, with subgroup XIIa (shown as orthogroup 6 in Fig. 3d) and subgroup XIIb harbouring the highest global expansion rates in oak. That is, 102 copies for subgroup XIIa and 50 copies for subgroup XIIb, corresponding to an expansion rate of 11.3-fold and 12.5-fold, respectively. Subgroup XIIa (containing, for example, flagellin-sensitive 2 (FLS2), EF-TU receptor (EFR) and Xa21) and subgroup XIIb (containing Xooinduced kinase 1 (XIK1), for example) included receptors known to play a role in the response to bacterial infections ${ }^{26}$. The orthogroups expanded in oaks also presented a significantly $\left(P<2 \times 10^{-16}\right)$ higher $\pi_{0} / \pi_{4}$ ratio than contracted or stable orthogroups (Supplementary Table 9). Moreover, the efficacy of purifying selection was remarkably low for the NB-LRR and RLK gene families, with mean $\pi_{0} / \pi_{4}$ ratios of 0.68 and 0.58 , respectively (Supplementary Note 4.1 ).

The enrichment of gene families relating to receptor-mediated signalling in oak led us to investigate whether similar enrichment had occurred in other trees. To this end, we compared trees and herbaceous species among the 16 plant genomes investigated. In eudicots, each distinct tree lineage provides an independent evolutionary experiment for investigating the genomic features relating to the tree lifestyle ${ }^{27}$. We found that 126 of the 36,844 orthogroups had undergone tree-specific expansion (Fig. 4b, Supplementary Data Set 7). These orthogroups were enriched in 61 GO terms, 


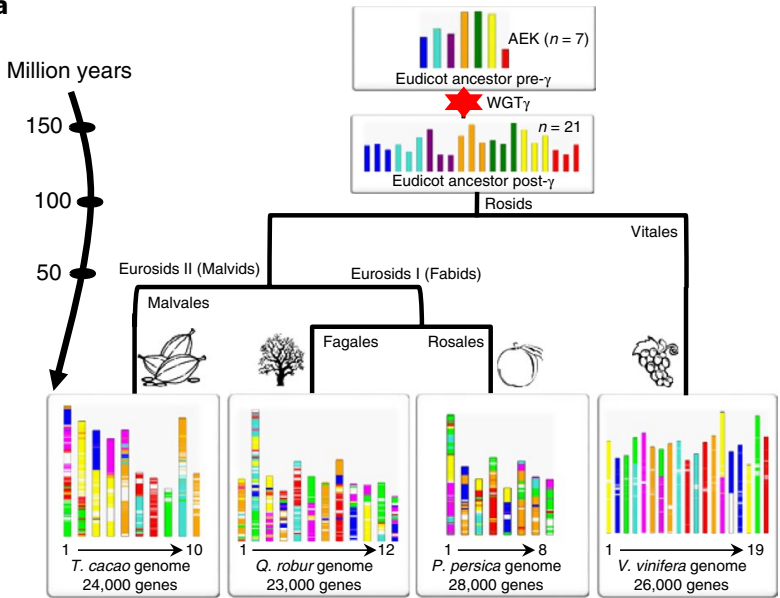

C

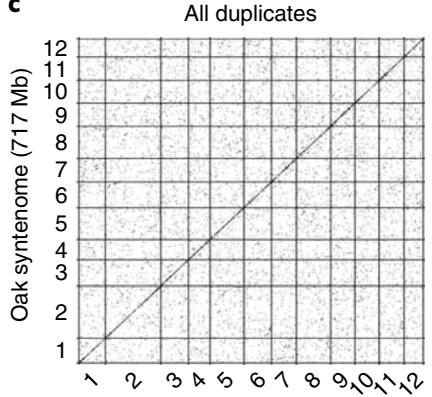

Oak syntenome (717 Mb)

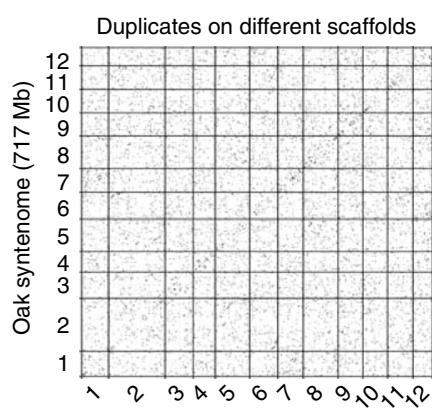

Oak syntenome $(717 \mathrm{Mb})$

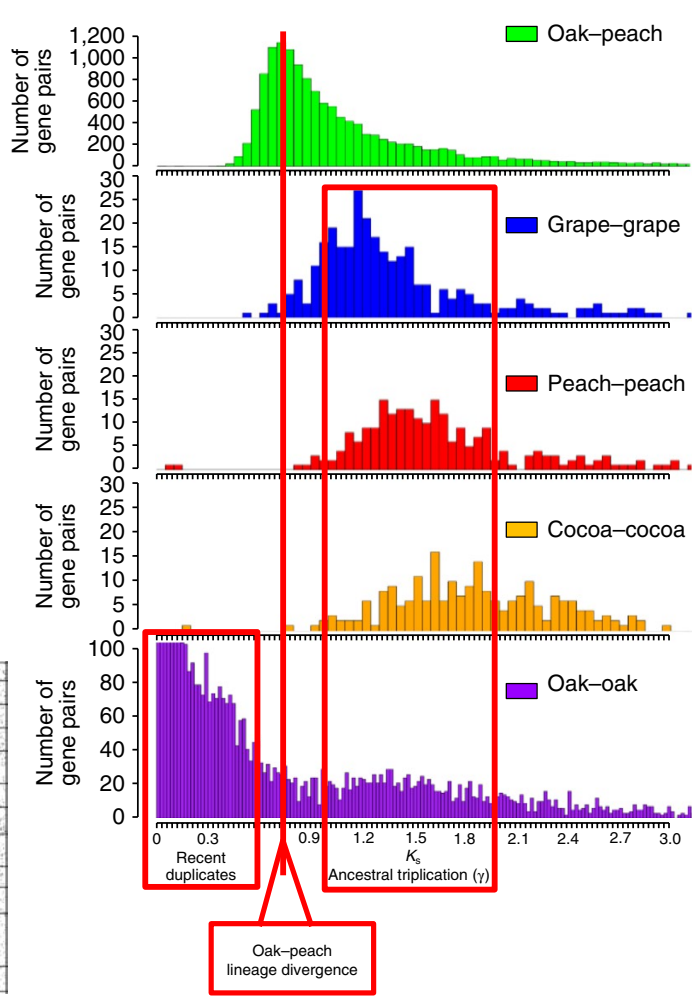

d
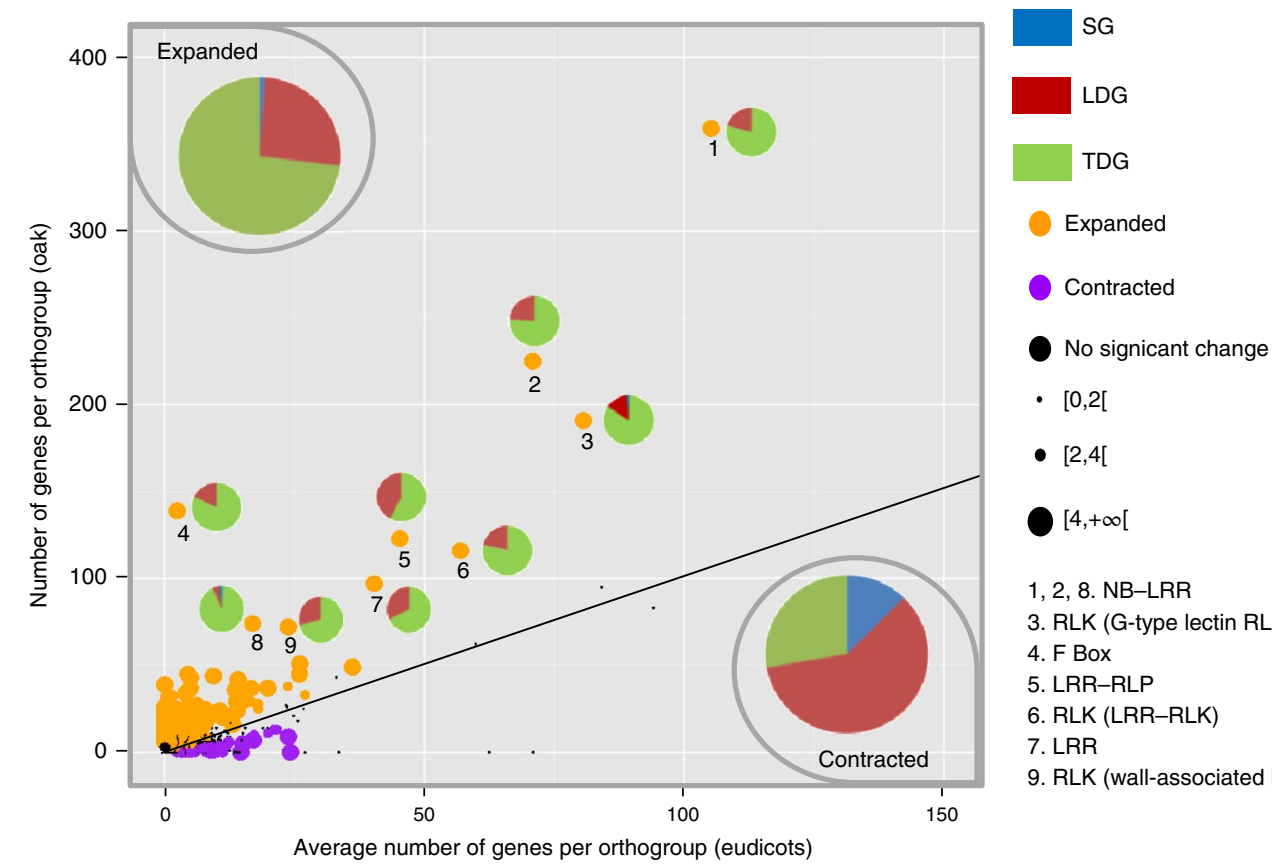

1, 2, 8. NB-LRR

3. RLK (G-type lectin RLK)

4. F Box

5. LRR-RLP

6. RLK (LRR-RLK)

7. LRR

9. RLK (wall-associated kinase)

Fig. 3 Evolutionary history of the oak genome. a, Evolutionary scenario of oak from the AEK of 21 (post- $\gamma$ ) and 7 (pre- $\gamma$ ) protochromosomes reconstructed from a comparison of the Vitales (grape), Rosales (peach) and Malvales (cocoa) major subfamilies. The modern genomes (bottom) are illustrated with different colours reflecting the seven ancestral chromosomes of AEK origin (top). WGT (red star) refers to the whole-genome triplication $(\gamma)$ shared among the eudicots. $\mathbf{b}, K_{\mathrm{s}}$ distribution of gene pairs for oak-peach orthologues as well as the shared $\gamma$ triplication in grape, peach, cocoa and oak. $K_{\mathrm{s}}$ distribution of all gene pairs in oak illuminate gene pairs from the $\gamma$ triplication as well as recent duplicates. $\mathbf{c}$, Dot plot representation of the oak genome against itself for the complete set of paralogous pairs (left) and without TDGs (right) representing the disappearance of the diagonal (TDGs) when low $K_{\mathrm{s}}$ values are removed. d, Expansion (524 orthogroups) and contraction (72 orthogroups) in oak relative to 15 other eudicot species. The pie charts reflect the contribution of TDGs, LDGs and singleton genes (SGs) to the significantly expanded and contracted orthogroups and to outstanding outliers (labelled 1-9). Numbers in square brackets associated with circle sizes stand for -log( $P$-value), computed from the oak branch-specific $P$-value provided by CAFE. 
a

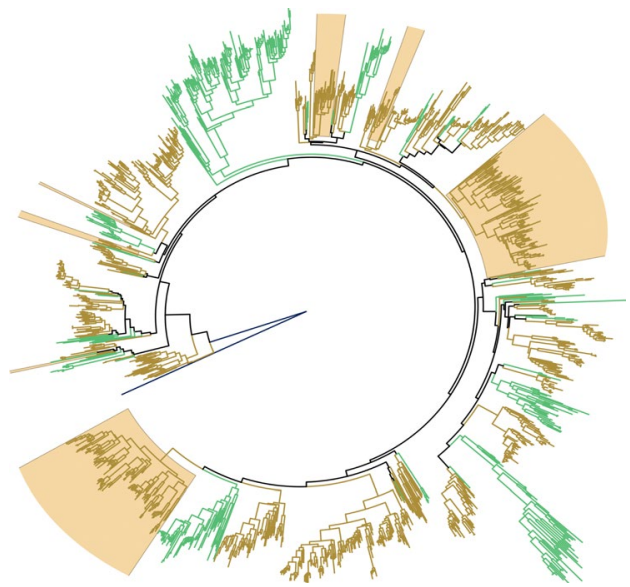

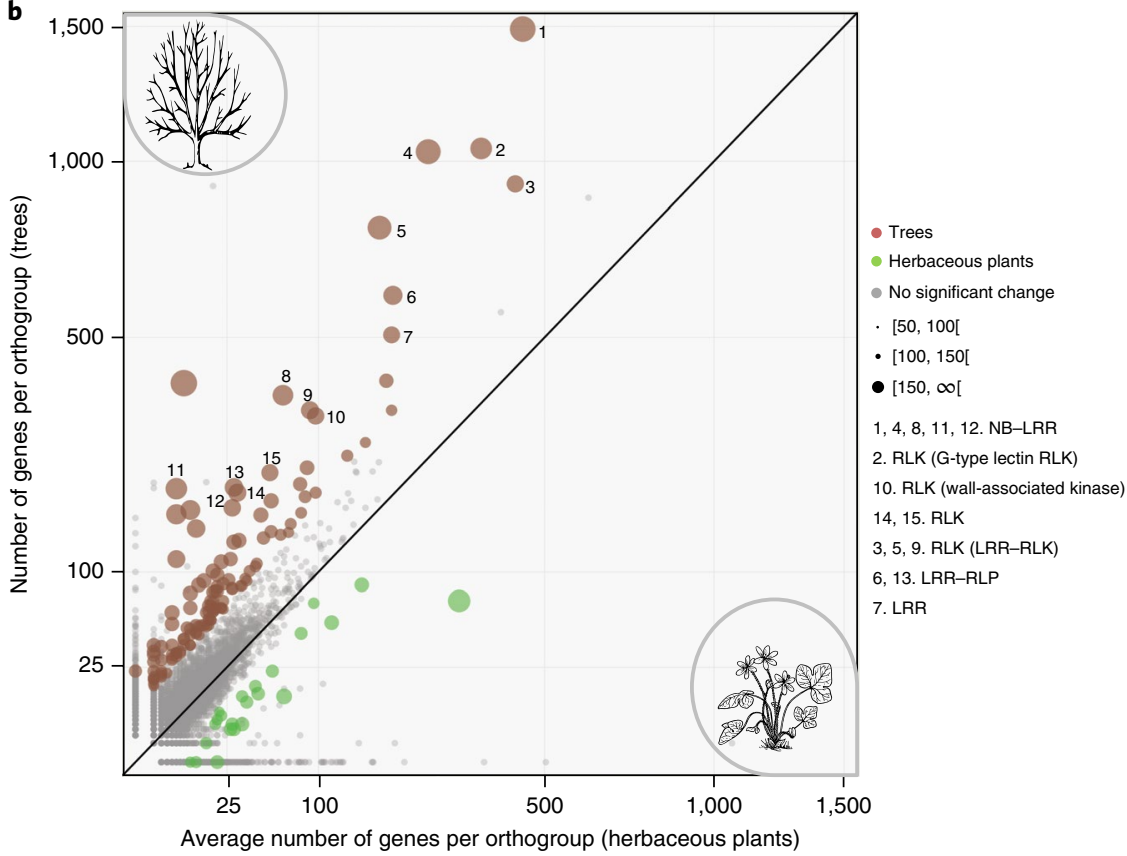

Fig. 4 | Expanded gene families in trees. a, Phylogeny of orthogroup 1 from Figs. 3d and 4b, established from the nucleotide-binding domains of 1,641 NB-LRR genes. Branches for trees and herbaceous species are shown in brown and green, respectively. Branches expanded in oak are shaded. For a higher resolution image see Supplementary Fig. 6. b. Scatter plot showing orthogroups expanded in trees and herbaceous plants (images from http://openclipart. org). Numbers in square brackets associated with circle sizes stand for -log( $P$-adjust), where $P$-adjust is the $P$-value of the binomial test adjusted for multiple testing.

largely (63\%) related to plant immunity (Supplementary Data Set 8, Supplementary Fig. 7). Ten of the 15 gene families displaying striking expansion in tree genomes (Fig. 4b) corresponded to NB-LRRs (orthogroups 1, 4, 8, 11 and 12), LRR-RLKs (orthogroups 3 (subgroup XIIb), 5 (subgroup XIIa) and 9) or LRR-RLPs (orthogroups 6 and 13). A phylogenetic analysis of the orthogroup most strongly expanded in trees (orthogroup 1 in Figs. $3 \mathrm{~d}$ and $4 \mathrm{~b}$ ) clearly highlighted the expansion of TIR-NB-LRRs in woody perennials relative to herbaceous species (Fig. 4a, Supplementary Fig. 6). Several TIR-NB-LRR genes from this cluster are involved in the perception of bacterial or oomycete pathogens in Arabidopsis (for example, $R p s 4$ or $R p p 5^{28,29}$ ). We also investigated the adaptive value of $\mathrm{R}$ genes within expanded orthogroups, making use of a recent metaanalysis of these membrane-bound receptor genes in 31 angiosperm genomes $^{25}$. We isolated 24 groups of oak lineage-specific expanded LRR-RLK paralogues and explored footprints of positive selection (Supplementary Data Set 9) based on the divergence between paralogous copies. In total, 19 groups $(80 \%)$ had a significant signature of positive selection, with similar proportions reported for only two other tree species (Malus, $73 \%$ and Populus, $87 \%$ ). We identified 260 sites subject to positive selection after the manual curation of protein sequence alignments in oak. More than $78 \%$ of these sites were located in LRR domains. As reported in a previous study ${ }^{25}$, positive selection mostly targeted four amino acids of the hypervariable region of the characteristic LXXLXLXX $\beta$-sheet/ $\beta$-turn structure of LRRs (Supplementary Fig. 8), which has been implicated in protein-protein interactions ${ }^{30}$. The high proportion of sites under positive selection in this domain therefore confirms the amino acid sequence diversification of these genes through fixation of amino acid changes.

In an opinion article ${ }^{31}$, it was suggested that the following three non-exclusive mechanisms could allow plants "to grow old without antibodies": numerous and highly diversified defence genes; favoured expansion of $\mathrm{R}$ gene families; and accumulation of somatic mutations, which can be transmitted to the next generation. Our study tackles all three genomic features that may contribute to the success of long-lived trees and finds support for all three suggested mechanisms.

In conclusion, we sequenced the oak genome and revealed its considerable genetic diversity, to which heritable somatic mutations may contribute. This work poses new research questions about the contribution of this mutational load in adaptation, in particular with regard to defences against new pests and pathogens. We also showed that the genome of this iconic tree went through a single paleohexaploidization event ( $\gamma$, shared among the eudicots), followed by a massive burst of recent local gene duplication. These duplications have amplified families of genes involved in defence against pathogens. We observed a parallel expansion of $\mathrm{R}$ generelated gene families across multiple tree species, suggesting that the immune system makes an essential contribution to the survival of long-lived plants over several centuries. The remarkable relaxation of purifying selection observed in oaks may facilitate the evolution of a richer and more diverse set of $\mathrm{R}$ genes, thereby conferring an advantage on these trees in their continuous arms race with pathogens $^{32}$. This dynamic is likely to apply particularly to oaks, with their remarkably long lifespan. However, the maintenance of such a diversity of R genes may be costly, and future studies should look at how trees control the expression of these immune receptors, through microRNA control, for example ${ }^{22}$.

\section{Methods}

Tree material. Pedunculate oak (Q. robur L., $2 n=2 \times=24)$ is an outcrossing, highly heterozygous diploid species. Flow cytometry analysis has shown that this species has a genome of $740 \mathrm{Mb}$ per $\mathrm{C}^{33}$, where the $\mathrm{C}$-value is the amount, in picograms, of DNA contained within a haploid nucleus. The " $3 \mathrm{P}$ " accession selected for establishment of the reference genome sequence for pedunculate oak is a tree of $\sim 100$ years of age located at the INRA Pierroton forestry research station (Aquitaine, France; $44^{\circ} 44^{\prime} \mathrm{N}, 00^{\circ} 46^{\prime} \mathrm{W}$ ). This tree has already been characterized at the genetic ${ }^{34,35}$ and genomic ${ }^{36,37}$ levels. The tree (used as a female parent) has also 
been crossed with accession A4 (used as a male parent) to generate a full-sibling progeny for studies of the genetic architecture of quantitative traits ${ }^{38-47}$. A graft copy of 3P was placed in darkness in July 2009, to trigger the release of as much starch as possible from second-flush leaves, in an in-house procedure that has been shown to improve the quality of DNA extraction from oak leaves. We harvested $140 \mathrm{~g}$ of etiolated leaves and stored them at $-80^{\circ} \mathrm{C}$ before DNA extraction.

DNA sample preparation for reference genome sequencing. An Invisorb Spin Plant Mini Kit (Stratec Molecular) was used to isolate genomic DNA and prepare short-read libraries for the Roche- 454 and Illumina sequencing platforms. DNA concentrations were determined using a Quant-iT dsDNA Assay Kit (Life Technologies) and a Qubit Fluorometer (Invitrogen). We checked the integrity of the genomic DNA by agarose gel electrophoresis and pulsed-field gel electrophoresis. Agarose-embedded high-molecular weight (HMW) DNA was prepared as described previously ${ }^{48}$, and modified as described previously ${ }^{49}$, to construct Illumina TruSeq Synthetic Long Read (TSLR) libraries. Agarose gel plugs were washed three times in Tris EDTA buffer and subjected to digestion with $8 \mathrm{U}$ o $\beta$-agarase (New England Biolabs) for $12-16 \mathrm{~h}$ at $42^{\circ} \mathrm{C}$. HMW DNA was then dropdialysed for $2.5 \mathrm{~h}$. DNA concentrations were quantified with the Quant-iT dsDNA Assay Kit. DNA quality was then checked using an Argus Qcard Kit (OpGen) and was estimated at $20-100 \mathrm{~kb}$

Sequencing. We prepared 454 single-end read libraries according to the standard procedure provided by Roche, with RL adaptors (GS FLX Titanium Rapid Library Preparation Kit; Roche Diagnostic). The libraries were sequenced with titanium chemistry on a 1/2 Pico Titer Plate on a 454 GS FlX instrument (Roche Diagnostic).

Illumina overlapping and tightly sized paired-end libraries were prepared using a semi-automated protocol. Briefly, genomic DNA (250 ng) was sheared using a Covaris E210 instrument (Covaris) to generate fragments of $150-400 \mathrm{bp}$ or $200-800 \mathrm{bp}$ in size for the overlapping and tightly sized libraries, respectively. End repair, A-tailing and ligation with Illumina-compatible adaptors (Bioo Scientific) were performed using a SPRIWorks Library Preparation System and a SPRI-TE instrument (Beckmann Coulter) according to the manufacturer's protocol. We selected fragments of 200-400 bp or 300-600 bp in size for the overlapping and tightly sized libraries, respectively. DNA fragments were then amplified by 12 cycles of PCR with Pfx Platinum Taq polymerase (ThermoFisher) and Illumina adapter-specific primers. We selected amplified library fragments of $\sim 300 \mathrm{bp}$ in size by electrophoresis in a $3 \%$ agarose gel for the overlapping libraries. For tightly sized libraries, fragments of $\sim 600 \mathrm{bp}$ in size were selected by electrophoresis in a $2 \%$ agarose gel.

The 3-kb mate-pair library was prepared according to the initial Illumina protocol (Illumina Mate Pair Library Kit), with $\sim 10 \mu$ g of genomic DNA subjected to Covaris fragmentation in the first step. The other mate-pair libraries were prepared using a Nextera Mate Pair Sample Preparation Kit (Illumina). Briefly, genomic DNA $(4 \mu \mathrm{g})$ was simultaneously fragmented by enzymatic treatment and tagged with a biotinylated adaptor. The resulting fragmented and tagged (tagmented) DNA was subjected to size selection $(3-5,5-8$ and $8-11 \mathrm{~kb})$ by regular gel electrophoresis, and circularized by overnight incubation with a ligase. Linear, non-circularized fragments were digested and circularized DNA was fragmented to generate fragments of 300-1,000 bp in size with the Covaris E210 system. Biotinylated DNA was immobilized on streptavidin beads, end-repaired, then $3^{\prime}$ -adenylated, and Illumina adapters were added. DNA fragments were amplified by PCR with Illumina adapter-specific primers and purified.

All Illumina library traces were evaluated using an Agilent 2100 Bioanalyzer (Agilent Technologies) and quantified by quantitative PCR using a KAPA Library Quantification Kit (KapaBiosystems) on a MxPro instrument (Agilent Technologies). Libraries were then sequenced as described in Supplementary Table 1.

Finally, 39,092 BACs (corresponding to a physical coverage of $3.5 \times$, Supplementary Note 2.1) were end-sequenced with dye terminator chemistry using an ABI 3730 sequencer (Applied Biosystems) as described previously ${ }^{50}$ The sequences can be obtained from GenBank (accession numbers HN154083HN174138, JS673272-JS676292, JS676293-JS684825 and FO926004-FO981373).

We prepared 14 libraries (Supplementary Table 1) from 5 different extracted HMW DNA samples with TSLR technology (previously known as Moleculo) according to the Illumina protocol. Briefly, genomic DNA (500 ng) was sheared into fragments of approximately $10 \mathrm{~kb}$ in size with g-Tube (Covaris). The fragments were subjected to end repair, A-tailing and adaptor ligation, and the ligated products were size-selected by gel electrophoresis to obtained fragments of 8-10 kb in size, which were quantified by qPCR. The long-insert library was then diluted such that each well of a 384-well plate contained $3 \mathrm{fg}$ of the library. The diluted products were subjected to long-range PCR, tagmentation and barcoding with 384 different barcoding PCR primers. The 384 barcoded libraries were pooled, purified and subjected to size selection. Each library was sequenced by 100 or 150 baselength read chemistry instrument (Illumina).

Sequence processing. Raw Roche/454 reads were used for subsequent analyses without processing. Illumina paired-end and mate-pair reads were cleaned in the following three-step procedure: sequencing adapters and low-quality nucleotides (quality value $<20$ ) were removed; sequences between the second unknown nucleotide $(\mathrm{N})$ and the end of the read were removed; and reads shorter than 30 nucleotides after trimming were discarded, together with reads and their mates mapping onto run quality control sequences (PhiX genome). The TSLRs were generated using the BaseSpace workflow. The primary sequencing data were then uploaded without modification to the BaseSpace cloud and processed using the standard Illumina workflow to generate long synthetic reads.

Genome size estimation by k-mer analysis. Before assembly, we analysed the $\mathrm{k}$-mer distribution of Illumina 100-bp paired-end reads (two lanes representing 95-fold coverage of the haploid genome) to obtain an independent estimate of the haploid size of the oak genome. The 31-mer distribution was generated using Jellyfish $^{51}$ (with the following parameters: -m 31 -s 2048M -C) and was uploaded to the GenomeScope website (http://qb.cshl.edu/genomescope/). We obtained an estimated haploid genome size of $736 \mathrm{Mb}$ (Supplementary Fig. 25), a value close to the $740 \mathrm{Mb}$ estimated by flow cytometry ${ }^{33}$.

Genome assembly. We first assembled the longest reads together (obtained from 454 and Moleculo libraries) to maximize the separation of the two haplotypes of accession $3 \mathrm{P}$ and to overcome the high level of heterozygosity. We used Newbler and Celera ${ }^{52}$ as the overlap-layout-consensus (OLC) assemblers. We used Newbler software (version MapAsmResearch-04/19/2010-patch-08/17/2010) with default parameters, with the addition of the -large and -sio options. As Newbler does not accept reads longer than $2 \mathrm{~kb}$, we split Moleculo reads into overlapping 1,999-bp fragments (with overlaps of 1,499 bp) and retained the origin of each fragment for further analysis (see next section). We obtained an assembly (named A1 in Supplementary Table 10) of 300,113 contigs with an N50 of $9.3 \mathrm{~kb}$ and a cumulative size of $1.31 \mathrm{~Gb}$, corresponding roughly to the size of the two haplotypes. We ran Celera with the following parameters: unitigger= bogart; merSize $=31$; merThreshold $=$ auto $^{\star} 2$; ovlMinLen $=800$; obtErrorRate $=0.03$; obtErrorLimit $=4.5$; ovlErrorRate $=0.03$; utgErrorRate $=0.015$; utgGraphErrorRate $=$ 0.015; utgGraphErrorLimit $=0$; utgMergeErrorRate $=0.03$; batThreads $=20$; utgMergeErrorLimit $=0$. This process produced an assembly (named $\mathrm{C} 1$ in Supplementary Table 11) composed of 29,255 contigs with an N50 of $9.5 \mathrm{~kb}$ and a cumulative size of $1.31 \mathrm{~Gb}$. The Celera assembler allows the direct input of raw Moleculo reads and we performed the scaffolding (that is, ordering and orienting of contigs) step directly on the Celera contigs of the $\mathrm{C} 1$ assembly.

Use of long reads to simplify the contig graph. Once the initial Newbler assembly was obtained, we used long-range information from Moleculo reads to simplify the contig graph. The Newbler output file "454ContigGraph.txt" describes the contig graph, in which the nodes are contigs and the edges are links between two contigs spanned by a read. Contigs were generally fragmented due to the presence of repeat or heterozygous regions. We extracted links between the contigs created from different parts of single long reads. Finally, a file containing all the links was generated (in DE format) and used as input for the string graph assembler (SGA) scaffolding module ${ }^{53}$. We obtained an assembly (named A2 in Supplementary Table 10) composed of 198,695 contigs with a N50 of $16.2 \mathrm{~kb}$ and a cumulative size of $1.33 \mathrm{~Gb}$

Scaffolding step. We used Illumina paired-end and mate-pair libraries to organize contigs and to produce scaffolds. We ran three iterations of the SSPACE scaffolder ${ }^{5}$ with the parameters $-\mathrm{k} 5$ and -a 0.7 , using the following libraries, ranked by increasing fragment size: 400 -bp paired-end, 3-kb mate-pairs, 5-kb mate-pairs and 8-kb mate-pairs. We then ran SSPACE again, with -k 2 and -a 0.7, using the Sanger BAC-ends and the previously scaffolded assembly. Sanger reads were transformed into Illumina-like reads by selecting the 100-bp window with the highest quality according to Sickle software ${ }^{55}$. We obtained two assemblies (A3 and C2 in Supplementary Tables 10 and 11, respectively). The most contiguous of these assemblies (A3) consisted of 9,025 scaffolds with an N50 of $818 \mathrm{~kb}$ and a cumulative size of $1.45 \mathrm{~Gb}$ (including $11.19 \%$ ambiguous bases).

Choice of the final assembly. The choice of the final assembly was based on the metrics of the two assemblies obtained with Celera and Newbler (assemblies C2 and A3) and comparisons with high-quality BACs (see Supplementary Note 2.1.3 and examples in Supplementary Fig. 9). We chose the Newbler assembly because it better discriminated between the two haplotypes.

Gap filling. The scaffold gaps of the A3 assembly were closed with GapCloser software $^{56}$ and Illumina paired-end reads. As input, we used $95 \times$ coverage (of the haploid genome) of overlapping paired-end reads and $95 \times$ coverage (of the haploid genome) of a standard paired-end library (400-600-bp fragments). We obtained an assembly (named A4 in Supplementary Table 10) consisting of 9,025 scaffolds with an N50 of $821 \mathrm{~kb}$ and a cumulative size of $1.46 \mathrm{~Gb}$ (including $4.63 \%$ ambiguous bases).

Bacterial decontamination. SNAP gene finder ${ }^{57}$ was applied to the entire assembly for draft gene prediction. We used an optimized calibration of SNAP based on the genewise alignment of $P$. persica coding sequences with the oak genome assembly. 
Predicted genes were then aligned against the NCBI NR database with BLAST-p. We kept the best match for each predicted protein and used the corresponding taxon. The 198 scaffolds containing $>50 \%$ bacterial genes for the assigned proteins were considered to be putative contaminants and were removed from the assembly file (assembly A5 in Supplementary Table 10).

Single-haplotype assembly. We used the Haplomerger v.1 pipeline ${ }^{58}$ to reconstruct allelic relationships in the released polymorphic diploid assembly and to reconstruct a reference haploid assembly. The diploid genome was first soft-masked with the following programs: $\mathrm{TRF}^{59}$ to mask tandem repeats; RepeatMasker ${ }^{60}$ to mask simple repeats, low-complexity and Viridiplantae-specific TEs; DUST ${ }^{61}$ to mask low-complexity sequences; and RepeatScout ${ }^{62}$ to mask unknown TEs. We then inferred a scoring matrix specific to the oak genome sequence, using $5 \%$ of the diploid assembly. The haploid genome was obtained from the soft-masked assembly and the specific scoring matrix with Haplomerger. We used the "selectLongHaplotype $=1$ " parameter to maximize gene content as recommended in the Haplomerger documentation, as we knew this would generate frequent switches between haplotypes (Supplementary Fig. 11). We also prevented Haplomerger from creating false joins between scaffolds by using external information. We used the genetic linkage map (see Supplementary Note 2.3) and prevented Haplomerger from joining scaffolds from different linkage groups by modifying the "hm.new_scaffolds" file. We obtained an assembly (named H1, Supplementary Table 2) composed of 1,409 scaffolds with an N50 of 1,343 kb and a cumulative size of $814 \mathrm{Mb}$ (including $2.94 \%$ ambiguous bases). We halved the size of the assembly, while retaining a completeness of gene content (evaluated with BUSCO $^{63}$, similar to that of the diploid assembly, see Supplementary Table 2). The haploid scaffolds were aligned with BACs for visual inspection to determine the correctness of this final release (Supplementary Figs. 11, 12 and 13). A comparison with an existing heterozygous plant genome shows that our assembly ranks among the best for a series of metrics (number of contigs and scaffolds, scaffold N50 size; Supplementary Table 3). As introduced in Supplementary Note 2.3, a chromosome scale genome was finally established using a high-density linkage map based on SNP markers ${ }^{8}$. We assessed the linear association between the genetic and physical positions of the SNPs using Spearman rank correlation.

Detection and annotation of transposable element. The REPET pipeline (http:// urgi.versailles.inra.fr/Tools/REPET) was used for the detection, classification $\left(\right.$ TEdenovo $^{64,65}$ ) and annotation (TEannot ${ }^{66}$ ) of TEs. The TEdenovo pipeline detects TE copies, groups them into families and defines the consensus sequence for each family containing at least five copies. The TEannot pipeline then annotates TEs using the library of consensus sequences.

The TEdenovo pipeline was used to search for repeats in contigs longer than $29,034 \mathrm{bp}$ ( $50 \%$ of the genome) from the first diploid version (V1) of the $Q$. robur reference genome sequence ${ }^{50}$. The first step used Blaster with the following parameters: [identity $>90 \%$, HSP (high-scoring segment pairs) length $>100 \mathrm{bp}$ and $<20 \mathrm{~kb}$, e-value $\leq 1 \mathrm{e}-300]$. The HSPs detected were clustered using Piler ${ }^{67}$, Grouper $^{66}$ and Recon ${ }^{68}$. Multiple alignments (with MAP ${ }^{69}$ ) of the 20 longest members of each cluster ( $n$ clusters) containing at least 5 members were used to derive a consensus. Consensus sequences were then classified on the basis of their structure and similarities relative to Repbase Update (v.17.11) ${ }^{70}$ and PFAM domain library v. $26.0^{71}$, before the removal of redundancy (with Blaster + Matcher as in the TEdenovo pipeline). Consensus sequences with no known structure or similarity were classified as 'unknown'.

The library of 4,552 classified consensus sequences provided by the TEdenovo pipeline was used to annotate TE copies throughout the genome with the TEannot pipeline. Three methods were used for annotation (Blaster, Censor and RepeatMasker). The resulting HSPs were filtered and combined. Three methods (TRF, Mreps and RepeatMasker) were also used to annotate simple sequence repeats (SSRs). TE annotation covered only by SSRs were then removed. Finally a "long join procedure" 72 was used to address the problem of nested TEs. This procedure finds and connects fragments of TEs interrupted by other more recently inserted TEs to build a TE copy. The nesting patterns of such insertions must respect the following three constraints: fragments must be collinear (both in the genome and with the same reference TE consensus sequence); of the same age; and separated by a more recent TE insertion. The percentage identity to the reference consensus sequence was used to estimate the age of the copy. Using the results of this first TEannot pipeline, we filtered out 2,047 consensus sequences that did not have a full-length copy in the genome. A copy may be built from one or more fragments joined by the TEannot long join procedure. We then performed manual curation to improve the TE annotation. We removed TE copies with consensus sequences identified as part of the host gene. These consensus sequences were built from a family of repeats containing at least five members and were classified as unknown by the TEdenovo pipeline. They were predicted to be host genes from multigene families. We also filtered out consensus sequences identified as chimeric. We obtained a final library of 1,750 consensus sequences, which together captured $52 \%$ of the oak genome, a value in the upper range of the values previously reported for plants.

Gene prediction and functional annotation of protein-encoding genes. We used EuGene v.4.0 $0^{73}$ to predict gene structure. EuGene predicts gene models from a combination of several lines of in silico evidence (ab initio and similarity). The EuGene pipeline was trained on a set of 342 genomic and full-coding complementary DNA pairs for which coding sequences were confirmed by protein evidence. One-third of the dataset was used for training the following $\mathrm{ab}$ initio gene structure prediction software: Eugene_IMM ${ }^{74}$, which is based on probabilistic models for discriminating between coding and non-coding sequences; SpliceMachine ${ }^{75}$, which was used to predict coding sequence (CDS) start and intron splicing sites; and FGENESH, an ab initio gene finder (http://linuxl. softberry.com/berry.phtml), which was used with Populus trichocarpa parameters. Another one-third of the dataset was used to optimize the EuGene parameters. The final one-third of the training dataset was used to calculate the accuracy of EuGene predictions. Sensitivity values of $85.8 \%$ and $75.2 \%$, and specificity values of $87.7 \%$ and $74.6 \%$, for exons and genes, respectively, were estimated.

We refined alignments with nucleotide similarity-based methods (Blat and Sim4) using transcript contigs from Q. robur and Quercus petraea ${ }^{76}$. We ensured that alignment quality was high by respecting the following criteria: $100 \%$ coverage and $98 \%$ identity for alignments with contigs shorter than $300 \mathrm{bp} ;<98 \%$ coverage and $98 \%$ identity for alignments with contig lengths between 300 and $500 \mathrm{bp} ;<95 \%$ coverage and an identity of $98 \%$ for alignments with contigs longer than $500 \mathrm{bp}$; and $<95 \%$ identity for all other cases. We also used BLAST-x 2.2.29+ to match protein sequences with sequences in protein databases, such as SwissProt, and databases built for species phylogenetically related to oak, such as P. persica v.1.39, Vitis vinifera v.1.45, P. trichocarpa v.2.10, Eucalyptus grandis v.2.01 and Arabidopsis thaliana v.1.67. We filtered out predicted genes overlapping TEs identified with the REPET package (see previous section), but retained TEs in introns and untranslated regions. The results of the various analyses were combined in EuGene to predict the final gene models. Predicted genes of $<100$ nucleotides in length were automatically filtered out by EuGene.

We initially predicted 77,043 protein-coding genes from the diploid version (V2) of the Q. robur genome sequence. In total, 2,067 genes from different gene families were manually curated by experts (Supplementary Note 3.5). From the 77,043 predicted genes, 43,240 were entirely recovered in the haplome, including 1,176 of the manually curated genes. Genes were tagged as 'unreliable' if their coding sequences were $<500$-bp long (corresponding to 166 amino acids), transcript coverage was $<90 \%$ or the genes were not curated manually. Based on these criteria, 13,575 genes were tagged as unreliable, and the remaining genes were tagged as 'regular' (28,484 genes) or 'manual' ( 1,176 genes).

We then performed a manual analysis of the 43,240 candidate gene models, guided first by an OrthoMCL run of the 16 genome sequences used in the evolutionary analysis (see the section "Oak karyotype evolution and genome organization"), in which we filtered out genes from OrthoMCL clusters associated with the following criteria: domains identified as plant mobile element domains (PMD domain) or TE domains (for example, transposases or GAG, a structural protein for virus-like particles within which reverse transcription takes place); and similarity to TE proteins, based on BLAST analyses against KEGG library results. We also checked that the OrthoMCL clusters contained $>90 \%$ Q. robur genes (that is, with only a minor contribution from other species) as follows: we filtered out 'potential pseudogenes' or small gene fragments predicted in regions of dubious assembly due to a high repeat content (that is, presence of TEs or repeated motifs in genes, such as NBS-LRR); we also filtered out unreliable and regular singletons (single genes not clustered with OrthoMCL) with a CDS $<500$ bp. Some small genes were classified as regular, as they were sufficiently covered by mRNA contigs, but they could be mapped to multiple sites within the genome and could not therefore be considered specific for the gene tagged.

Automated functional annotation was performed on the 25,808 predicted proteins (listed in Supplementary Data Set 1), using an in-house pipeline (FunAnnotPipe), mostly largely on the InterProScan v.5.13-52.077 webservice for domain and motif searches. This included all the manually curated genes, $78 \%$ of the regular set and $17 \%$ of the unreliable set. Subcellular targeting signals and transmembrane domains were predicted with SignalP, TargetP and TMHMM ${ }^{78}$ and InterProScan. We also carried out similarity searches with BLAST-x V2.2.29+ against PDB, Swissprot and $\mathrm{KEGG}^{79}$, and rpsBLAST (14 June 2009) searches for conserved domains against the CDD database ${ }^{80}$ and $\mathrm{KOG}^{81}$. We also used the BLASTKoala webservice (http://www.kegg.jp/blastkoala/, January 2016) to associate KEGG orthology groups, and E2P2 to identify the associated enzyme codes when relevant (https://dpb.carnegiescience.edu/labs/rhee-lab/ software, v.3.0).

We assigned 'definitions' to the predicted proteins as proposed by Phytozome ${ }^{82}$ and D. M. Goodstein (personal communication). We used the annotation from the most accurate analysis as input: EC number (E2P2), KEGG orthology group (KO; KEGGKOALA), PANTHER (InterProScan), KOG (conserved domain database for eukaryotic organisms) and PFAM (InterProScan). We then calculated the multiplicity $(\mathrm{M})$ of annotations across the entire genome, both as single (for example, KOG0157, PF0064 and PF0005) and same-type compound keys (for example, PF0064//PF0005). Mixed compound keys were not considered (for example, KOG0157//PF0064). Weighting (W) factors were applied to protein definitions to give priority to the most informative annotations as follows: $\mathrm{EC}=1$, $\mathrm{KO}=1.1, \mathrm{PANTHER}=2, \mathrm{KOG}=3, \mathrm{PFAM}=4$. The final protein definition corresponds to the least frequent description (minimum $\mathrm{M} \times \mathrm{W}$ value) from this 
analysis. The key advantage of this approach is that it makes it possible to assign a protein definition without over-representing a single type of annotation found at multiple locations. As a result, a protein definition was assigned to $87 \%$ of the predicted oak proteins (Supplementary Data Set 1).

Estimation of heterozygosity of the reference genotype 3P. All the short Illumina paired-end reads used to produce the $3 \mathrm{P}$ oak reference genome were mapped against the haplome assembly with bowtie $2^{83}$, using standard parameters for the "fast end-to-end" mode. Duplicated mapped reads were removed with Picard (http://broadinstitute.github.io/picard/). SAMtools/bcftools ${ }^{84}$ were used to call variants. We then used a combination of custom-made scripts (available at http:// www.oakgenome.fr) to calculate coverage and estimated allele frequency from the "DP4" tag of the .vcf file. We discarded all SNPs with a minor allele frequency value $<0.25$ and all insertions and deletions; the proportion of heterozygous sites on the chromosomes was then calculated with a sliding window approach. For each window, this proportion was weighted by the $N \%$ and the fraction covered, defined here as the proportion of bases within a window satisfying the same sequence depth criteria as used for SNP calling.

Pool-seq-based estimator of oak genetic diversity. Branches from 38 pedunculate oak trees were sampled in spring 2011 from oak stands within the maritime pine forest (Supplementary Table 17, Supplementary Fig. 53) of the Landes (Southwest France). Branches were harvested with a telescopic pole pruner and placed in darkness for 3 days to trigger the release of starch from chloroplasts. Etiolated leaves were then harvested and their DNA was extracted using a DNeasy Plant Mini Kit according to the manufacturer's instructions (Qiagen). The amount of DNA was assessed using a NanoDrop ND-1000 spectrophotometer (NanoDrop Technologies Inc, Rockland, DE, USA) and DNA quality was assessed visually by electrophoresis in a $1.2 \%$ agarose gel. The 38 genotypes were genotyped with a 12-plex of expressed sequence tag SSRs and an 8-plex of genomic SSRs ${ }^{85}$. We estimated genetic relatedness between genotypes with COANCESTRY ${ }^{86}$, as described previously ${ }^{87}$, and the degree of introgression of sequences from sessile oak (Q. petreae) was assessed using STRUCTURE ${ }^{88}$, as described previously ${ }^{85}$. Following this analysis, we excluded three samples identified as possibly related and eight samples displaying a large degree of introgression from sessile oak. We then randomly selected 20 of the remaining 27 trees (Supplementary Table 18) for whole-genome sequencing by pool-sequencing (pool-seq) techniques ${ }^{89}$.

DNA from these 20 oaks was re-extracted from individual samples using an Invisorb Spin Plant Mini Kit (Stratec Molecular). We visually checked the DNA quality by gel electrophoresis (1.5\% agarose) and estimated the concentration and purity using a NanoDrop 1000 spectrophotometer (NanoDrop Technologies). We then pooled DNA from individual samples to obtain an equimolar solution with a final concentration of $570 \mathrm{ng}^{-1}$. We used this pool of DNA to prepare a pairedend genomic library with a Paired-End DNA Sample Preparation Kit (Illumina). This library was sequenced on 10 lanes of a HiSeq2000 sequencer (Illumina) $(2 \times$ 100 -bp paired-end reads), generating $1,732,899,595$ paired-end reads $(331 \mathrm{~Gb}$, that is, $\sim 400 \times$ haploid genome coverage).

Raw reads were trimmed to remove low-quality bases, as described in the "Sequence processing" section. All reads were then mapped against the oak haplome assembly with bowtie $2^{83}$, using standard parameters for the "sensitive end-to-end" mode. Potential PCR duplicates were removed using Picard (http:// broadinstitute.github.io/picard/). Samtools ${ }^{84}$ and PoPoolation $2^{90}$ were then used to call SNPs with counts of at least 10 for the alternate allele and a depth between 50 and $1,000 \times$ at the position concerned. All SNPs with a minor allele frequency value $<0.05$ were discarded. After subsampling the pileup at all retained positions to a uniform coverage of $30 \times$ ("subsample-pileup.pl", PoPoolation suite ${ }^{91}$ ), we used the "variance-sliding.pl" script (PoPoolation ${ }^{91}$ ) to calculate $\pi$ along chromosomes by a sliding window approach (1-Mb sliding windows, 250-kb steps, Supplementary Figs. 4 and 15).

Estimate of genetic diversity and $\boldsymbol{\pi} \mathbf{0} / \boldsymbol{\pi} \mathbf{4}$ ratio. We estimated genetic diversity as pairwise nucleotide diversity $(\pi)$ at zerofold and fourfold sites for each proteincoding gene, as described previously ${ }^{9}$. We then defined the $\pi_{0} / \pi_{4}$ ratio as the ratio of mean $\pi_{0}$ to mean $\pi_{4}$ over all genes. We also computed these metrics on manually curated genes, which showed that the gene model quality did not compromise our findings. We compared estimates between genes from expanded, contracted and unchanged gene families (orthogroups) in oak. We accounted for the different gene family sizes by randomly sampling 1,000 genes from each of these three categories and repeating the operation 100 times.

Detection of somatic mutations. Our objective was to show that somatic mutations (in terms of SNPs) exist in a long-lived plant and transmitted to the next generation. Because we did not intend to provide a comprehensive estimate of the number of somatic mutations in the studied 100-year-old tree, it is meaningless to compare our result to an expected number of somatic mutations because of the following unknown factors: the substitution rate per site and per generation; the number and pattern of mitotic divisions from zygote and axillary buds; and cell death and bud abortion rates.
We investigated somatic mutations by resampling the $3 \mathrm{P}$ genotype used to sequence and assemble the reference genome, as described below.

Vegetative buds were collected from the extremities of three second-order branches of the 2011 increment in February 2012: two lateral branches (L1 and L2) and the tree apex (L3). We used dendrochronology (tree-ring dating) to date the time of initiation of the L1 and L2 branches (Supplementary Fig. 5). To this end, we collected 5-mm diameter wood cores from the insertion point of the selected branches with an increment borer. We also dated the age of the tree by taking a core just above ground level and counting the number of rings under a microscope. We estimated that the L1 and L2 branches had been initiated 15 and 47 years earlier, respectively, and that the terminal branch was at least 85 years old.

DNA was extracted from three sets of vegetative buds sampled at location L1, L2 and L3 using the Invisorb Spin Plant Mini Kit (Stratec Molecular). For each sample, six independent DNA extractions were carried out on a pool of buds. DNA quality was checked by electrophoresis in a $1.5 \%$ agarose gel. DNA concentration and purity were assessed with a NanoDrop 1000 spectrophotometer (NanoDrop Technologies). Individual DNA samples from the same branch were the pooled in an equimolar solution to obtain a final concentration of $769-1,388 \mathrm{ng}^{-1}$. We prepared tightly sized paired-end libraries (600 bp in size) as described in the "Sequencing" section and sequenced each of these libraries on one to four lanes of a HiSeq2000 or HiSeq2500 sequencer (Illumina) (Supplementary Table 19, 100-bp or 250-bp paired-end reads). We obtained 284-fold (L1), 250.5-fold (L2) and 264.9-fold (L3) haploid genome coverage for these samples. For each of the three branches (L1, L2 and L3), reads were mapped against the reference genome sequence with BWA-MEM ${ }^{92}$ using the default parameters, except for minimum seed length $(k=79)$. After sorting, PCR duplicates were removed with Picard (http://broadinstitute.github.io/picard/). We searched for somatic mutations using MuTect (a program developed for the detection of somatic point mutations in heterogeneous cancer samples ${ }^{11}$ ) to compare the three libraries (six pairwise combinations; Supplementary Table 20). This frequency-dependent detection approach was considered to be particularly well suited to identify somatic mutations in plants.

Because considering sequencing error (that is, false positives) is essential for detecting mutations and is vital for drawing valid conclusions, particularly with respect to the detection of somatic mutations within a single individual, we addressed this concern and took all possible actions to minimize it. Thus, the accuracy of somatic point mutations was ensured by considering only those sites with the following characteristics: a minimum depth of 50x in both the reference and potentially mutated libraries; no mutant (that is, alternative) allele in the reference library; and a minimum frequency of $20 \%$ for the mutant allele in the potentially mutated library (that is, each somatic mutation was supported by 10 alternative alleles or more). We then filtered out candidate somatic mutations by using a cross-validation procedure. Across all pairwise comparisons, we only kept somatic mutations with a temporal pattern coherent with the chronology of branch development (see Supplementary Table 20 for details). These multiple comparisons made it possible both to validate the detected mutations and to reconstruct their mutational history along the trunk or the two branches. Finally, we discarded 15 additional candidate mutations among the set of 61 reliable somatic mutations. Indeed, for this set of 15 somatic mutations, we recovered the same alternate allele in the pool of 20 pedunculate individuals (see the section "Pool-seq-based estimator of oak genetic diversity") at a frequency $>0.005$. Note that $\mathrm{f}($ alt $)<0.005$ remains a stringent criterion considering Illumina sequencing error calls (0.024). As a consequence, we cannot rule out that some true positives were excluded at this step. However, our objective was to be as conservative as possible in order to study the transmission of these somatic mutations to the next generation (Supplementary Table 5).

We studied the transmission of somatically acquired mutations to the offspring by extracting DNA using a DNAeasy 96-Plant Kit (Qiagen) from 116 acorns sampled from the extremities of the L1 and L2 branches (Fig. 2b). DNA was extracted after the dissection of embryonic tissues (radicle and plumule) from the acorn. We used 15 ng DNA to genotype the offspring using a MassArray iPLEX Assay (Agena Bioscience) according to the manufacturer's instructions. Primers were designed, and 33 SNPs were multiplexed in the Assay Design Suite (Agena Bioscience). Allele calling was processed in Typer Viewer v.4.0.26.75 (Agena Bioscience). This 39-plex assay contained 12 control SNPs and 21 candidate somatic mutations (Supplementary Table 5). Control SNPs were used to provide an estimate of the selfing rate likely to impair interpretation of the segregation of somatic mutations in the offspring. The control SNPs were loci homozygous in the reference genotype $3 \mathrm{P}$ and found at a very low frequency in the pool of 20 pedunculate oaks; that is, with minimum allele frequencies ranging from 0.02 to 0.05 . Embryos resulting from the self-pollination of $3 \mathrm{P}$ were expected to be homozygous for the reference allele, and most outcrossed embryos were expected to be heterozygous. We observed a mean heterozygosity of 0.54 over the 12 control loci. In the absence of selfing and based on allele frequencies estimated in the pool of 20 individuals, mean heterozygosity would have been close to 0.96 , thus suggesting a relatively high rate of selfing (44\%). Unamplified loci $(2 / 21$ SNPs; Supplementary Table 6) were excluded from the analysis. The overall rate of missing data was high (39\% for missing somatic mutations and $54 \%$ for control 
SNPs), so all polar plots from Typer Viewer software of the MassArray iPLEX assay were inspected visually to check that genotyping calls were accurate.

Oak karyotype evolution and genome organization. We used two previously defined parameters ${ }^{93}$ to increase the stringency and significance of BLAST sequence alignment by either parsing BLAST results and rebuilding HSPs or using pairwise sequence alignments to identify accurate paralogous relationships within oak (25,808 gene models; Supplementary Data Set 1). Orthologous relationships between oak and grape $\left(26,346\right.$ genes on 19 chromosomes $\left.^{15}\right)$, peach $(28,086$ genes on 8 chromosomes $\left.{ }^{16}\right)$ and cocoa $\left(23,529\right.$ genes on 10 chromosomes $\left.^{17}\right)$ were also determined. We estimated the sequence divergence of paralogues and orthologues from the $K_{\mathrm{s}}$ calculated with the PAML 4 package ${ }^{94}$ for oak-peach, grape-grape, peach-peach, cocoa-cocoa and oak-oak gene pairs. Dot plot representations of synteny and paralogy were obtained with the R package ggplot2 (http://ggplot2. org/; Supplementary Fig. 16).

Gene family expansion and contraction in oak. A classification of groups of orthologous sequences (orthogroups, also referred to here as gene families or clusters) was developed for 16 eudicot plant species: all the predicted oak proteins (corresponding to 25,808 gene models) and the proteins catalogued from 15 other eudicot species (Supplementary Table 21, Supplementary Note 5.1.2). The other eudicot species were Arabidopsis lyrata, A. thaliana, Citrus clementina, Carica papaya, E. grandis, Fragaria vesca, Glycine max, Malus domestica, P. persica, $P$. trichocarpa, Ricinus communis, Solanum tuberosum, Theobroma cacao, V. vinifera (genomes available from https://phytozome.jgi.doe.gov) and Citrullus lanatus (genome available from http://www.icugi.org/cgi-bin/ICuGI/index.cgi). These 15 plant genomes were selected on the basis of the following criteria: availability of genome sequences and gene models from public databases; assembly quality (N50 length of assembled fragments) and the number of predicted genes; classification (order, family and genus), the main goal being to cover the entire range of eudicots. The classification was based on a BLAST-p all-against-all comparison of the complete proteomes $\left(E-v a l u e ~<10^{-5}\right.$ ) of these species, followed by clustering with OrthoMCL $2.0 .9^{95}$ using default parameters. GO terms for 15 of the plant proteomes were retrieved from Phytozome. For watermelon, the CDS were downloaded from the following website: http://cucumber.genomics.org.cn/ page/cucumber/index.jsp. We used Interproscan ${ }^{96}$ to assign GO terms. GO term enrichment analysis was then carried out on the expanded orthogroups in oak (Supplementary Note 5.4).

We then used CAFE v.3.1 $1^{97,98}$ with phylogenetic tree information (drawn from http://etetoolkit.org/treeview/) derived from previous studies ${ }^{18}$ (Supplementary Fig. 17) to identify the orthogroups displaying expansion and contraction in oak using a $P$ value threshold of 0.01 .

Identification and validation of TDGs in oak. Duplicated genes in oak were identified from the $K_{\mathrm{s}}$ paralogue distribution (see purple $K_{\mathrm{s}}$ distribution in Supplementary Fig. 20) and are illustrated in the dot blot shown in Supplementary Fig. 21 (see also Supplementary Note 5.2). We extracted duplicated genes from the complete repertoire of paralogues and generated pairwise alignments of protein sequences with BLAST-p and filters based on alignment identity and length (CIP (cumulative identity percentage)/CALP (cumulative alignment length percentage $=50 \% / 50 \%)$. Then we sorted protein sequences by their coordinates on each of the 12 oak chromosomes. We defined TDGs as duplicates separated by up to three genes and LDGs as duplicates separated by more than three genes. The remaining genes were classified as singleton genes.

We checked that these recent TDGs in oak were true duplicates rather than different alleles or duplication artefacts arising during haplome construction (during the scaffolding or merging steps of our hierarchical assembly pipeline) by applying two verification procedures based on sequence variation and sequence coverage. First, we obtained pairwise nucleotide sequence alignments, using MUSCLE with standard parameters ${ }^{99}$, for all 9,189 putative TDGs. For each alignment, summary statistics were calculated with $\mathrm{AMAS}^{100}$. We found that 15 gene pairs involved in local duplications presented no gaps or polymorphisms and could be considered to be putative assembly artefacts. This corresponds to only a minor fraction $(0.13 \%)$ of the 11,695 pairwise alignments. In contrast, we found that 8,115 pairs of TDGs $(69.4 \%)$ displayed substantial sequence divergence (gap length $>10 \%$ and a proportion of variable sites $>2 \%$ ), greater than that between pairs of alleles (Supplementary Fig. 22). Indeed, from the 12,603 allelic pairs obtained by comparing the diploid and haploid versions of the oak genome sequence available for this comparison (indicated as 2:1 relationships in the last column of Supplementary Data Set 1), 1,278 (that is, 10.1\%) had a gap length > $10 \%$ and a proportion of variable sites $>2 \%$. Second, a per-base coverage analysis based on reads from the genes classified as TDGs, LDGs and singleton genes indicated that TDGs did not represent half the coverage of the other two categories (illustrated for the longest scaffold in Supplementary Fig. 23), ruling out the alternative hypothesis that TDGs are allelic regions or artefactual duplications due to errors in the assembly process.

Detection of significant expansion and contraction in woody perennials. Particular outcomes of gene family expansion and contraction may be associated with the lifestyle of a tree, but no study of differential gene gains and losses has been performed at the genomic scale in eudicots (Supplementary Note 5.3). We therefore applied an additional criterion when selecting the 15 plant species for comparative genomic analyses; that is, the growth habit (woody perennial versus herbaceous). The genomes of nine woody perennials and seven herbaceous species were available for the investigation of orthogroup expansion in woody species (trees). These two categories were homogeneous in terms of OrthoMCL orthogroups. For a range of variables, including the number of genes per orthogroup (Supplementary Fig. 24), the mean number of genes per orthogroup, the percentage of orthogroups with no genes, and the number of species-specific orthogroups (Supplementary Table 22), no statistical difference was found between the two categories.

We investigated whether a given orthogroup showed significant expansion or contraction in trees by comparing the total number of genes per orthogroup between the two types of growth habit. Given the relatively small number of species per category, we performed a binomial test with a probability of success of $p(W)=9 / 16$. From the initial set of 36,844 orthogroups, we retained orthogroups displaying a statistically significant outcome in terms of gene counts (false discovery rate-adjusted $P$ value $<0.05^{101}$ ). The minimal contribution to each category was set to five for trees and four for herbaceous species to minimize bias due to the number of species analysed. We found that 126 orthogroups were expanded (corresponding to 23,321 genes; that is, 155.1 genes per orthogroup on average) and 23 were contracted in woody perennials relative to herbaceous species. Functional identities and orthogroup sizes are presented for all significantly expanded or contracted orthogroups in Supplementary Data Set 7 (sheets 2 and 4). GO term enrichment analysis was carried out on the 126 expanded and 23 contracted orthogroups (see next section). We also identified a set of remarkable orthogroups (outliers in Fig. 3d), differing between trees and herbaceous species and including at least five genes in five different species.

GO enrichment analysis. All GO term enrichment analyses were performed using R 3.3.1 software ${ }^{102}$ and the topGO 2.22.0 package $^{103}$. The weight01 algorithm ${ }^{103}$ and Fisher's exact test were used to detect significant enrichment in GO terms in the various test sets. As stated by the authors of topGO, the $P$ value of a GO term is conditioned on the neighbouring terms. The tests are therefore not independent, and the multiple testing theory does not directly apply. $P$ values should therefore be interpreted as corrected or not affected by multiple testing.

Fold-enrichment was defined as illustrated below:

- At the gene level, if $52 / 9,189$ (that is, $0.56 \%$ ) of input genes are involved in "chitinase activity" and the background level is $60 / 25,808$ genes (that is, $0.23 \%$ ) associated with chitinase activity, the fold-enrichment is approximately $0.56 \% / 0.23 \%=2.43$ for this molecular function.

- At the orthogroup level, if $6 / 126$ (that is, $4.76 \%$ ) of input orthogroups are involved in "protein serine/threonine kinase activity" and the background level is $50 / 36,844$ orthogroups (that is, $0.136 \%$ ) associated with protein serine/threonine kinase activity, the fold-enrichment is approximately $4.76 \% / 0.136 \%=35$ for this molecular function

The first example corresponds to the fold-enrichment calculations performed for TDGs, LDGs, singleton genes and orthogroups expanded in oak. The second corresponds to the fold-enrichment calculation for orthogroups expanded in woody perennials.

Web resources. We set up several tools and a browser based on the international open-source project Generic Model Organism Database (http://www.gmod.org) to provide us with access to both structural information and functional annotation (Supplementary Note 6). WebApollo/JBrowse ${ }^{104}$ was set up (https://urgi.versailles. inra.fr/WebApollo_oak_PM1N/jbrowse/) and populated with the oak reference genome sequence (that is, 12 chromosomes comprising 876 scaffolds and 533 unassigned scaffolds) and 34 BAC sequences. Several tracks were superimposed on these sequences, including predicted genes, predicted TEs, predicted noncoding RNAs, proteins from several species, oak unigenes, RNA-sequencing data and quantitative trait loci. The 'chunk' track represents virtual contig sequences separated by $N$ stretches of no more than 11 consecutive bases. Intermine (v.1.3.9) ${ }^{105}$ was used to gather and make available all the information (structural and functional) produced for each protein-coding gene (https://urgi.versailles.inra. fr/OakMine_PM1N/begin.do). All the details about data sources are available from the application in the datasource panel. For JBrowse, tracks have been generated from the reference genome using data generated for EuGene prediction.

Reporting summary. Further information on experimental design is available in the Nature Research Reporting Summary linked to this article.

Code availability. The source code for the prediction of miRNA is available as a workflow at https://forgemia.inra.fr/genotoul-bioinfo/ngspipelines/tree/master/ workflows/srnaseq. Custom-made scripts for the estimation of heterozygosity of the reference genotype $3 \mathrm{P}$ are available at the oak genome website (http://www. oakgenome.fr/?page_id=587). 
Data availability. The oak haploid genome assembly and corresponding annotation have been deposited in the European Nucleotide Archive under project accession code PRJEB19898. Other sequence release data are indicated in Supplementary Tables 1, 13, 14 and 19, and Supplementary Data Set 10. Data (including intermediate genome assemblies, .vcf files used to detect somatic mutations and estimate heterozygosity) are available at the oak genome website hosted as a permanent resource by INRA (http://www.oakgenome.fr/).

Received: 12 November 2017; Accepted: 8 May 2018; Published online: 18 June 2018

\section{References}

1. Camus, A. Les Chênes: Monographie du Genre Quercus et Monographie du Genre Lithocarpus (P. Lechevalier, Paris, 1954).

2. Logan, W. B. Oak: The Frame of Civilization (W. W. Norton \& Company, New York, 2005).

3. Manos, P. S. \& Stanford, A. M. The historical biogeography of Fagaceae: tracking the tertiary history of temperate and subtropical forests of the Northern Hemisphere. Int. J. Plant Sci. 162, S77-S93 (2001).

4. Whitham, T. G. \& Slobodchikoff, C. N. Evolution by individuals, plant-herbivore interactions, and mosaics of genetic variability: the adaptive significance of somatic mutations in plants. Oecologia 49, 287-292 (1981).

5. Folse, H. J. \& Roughgarden, J. Direct benefits of genetic mosaicism and intraorganismal selection: modeling coevolution between a long-lived tree and a short-lived herbivore. Evolution 66, 1091-1113 (2012).

6. Pineda-Krch, M. \& Fagerström, T. On the potential for evolutionary change in meristematic cell lineages through intraorganismal selection. J. Evol. Biol. 12, 681-688 (1999).

7. Padovan, A. et al. Transcriptome sequencing of two phenotypic mosaic Eucalyptus trees reveals large scaletranscriptome re-modelling. PLoS ONE 10, e0123226 (2015).

8. Bodénès, C., Chancerel, E., Ehrenmann, F., Kremer, A. \& Plomion, C. High-density linkage mapping and distribution of segregation distortion regions in the oak genome. DNA Res. 23, 115-124 (2016).

9. Chen, J., Gl, S. \& Lascoux, M. Genetic diversity and the efficacy of purifying selection across plant and animal species. Mol. Biol. Evol. 34, 1417-1428 (2017).

10. Brown, C. L., Mcalpine, R. G. \& Kormanik, P. P. Apical dominance and form in woody plants : a reappraisal. Am. J. Bot. 54, 153-162 (1967).

11. Cibulskis, K. et al. Sensitive detection of somatic point mutations in impure and heterogeneous cancer samples. Nat. Biotechnol. 31, 213-219 (2013).

12. Schmid-Siegert, E. et al. Low number of fixed somatic mutations in a long-lived oak tree. Nat. Plants 12, 926-929 (2017).

13. Gill, D. E., Chao, L., Perkins, S. L. \& Wolj, J. B. Genetic mosaicism in plants and clonal animals. Ann. Rev. Ecol. Syst. 26, 423-444 (1995).

14. Murat, F., Armero, A., Pont, C., Klopp, C. \& Salse, J. Reconstructing the genome of the most recent common ancestor of flowering plants. Nat. Genet. 49, 490-496 (2017).

15. Jaillon, O. et al. The grapevine genome sequence suggests ancestral hexaploidization in major angiosperm phyla. Nature 449, 463-467 (2007).

16. The International Peach Genome Initiative et al. The high-quality draft genome of peach (Prunus persica) identifies unique patterns of genetic diversity, domestication and genome evolution. Nat. Genet. 45, 487-494 (2013).

17. Argout, X. et al. The genome of Theobroma cacao. Nat. Genet. 43, 101-108 (2011).

18. Salse, J. Ancestors of modern plant crops. Curr. Opin. Plant Biol. 30, 134-142 (2016).

19. Murat, F. et al. Karyotype and gene order evolution from reconstructed extinct ancestors highlight contrasts in genome plasticity of modern rosid crops. Genome Biol. Evol. 7, 735-749 (2015).

20. Li, Q. et al. Explosive tandem and segmental duplications of multigenic families in Eucalyptus grandis. Genome Biol. Evol. 7, 1068-1081 (2015).

21. Hanada, K., Zou, C., Lehti-Shiu, M. D., Shinozaki, K. \& Shiu, S.-H. Importance of lineage-specific expansion of plant tandem duplicates in the adaptive response to environmental stimuli. Plant Physiol. 148, 993-1003 (2008)

22. Zhang, Y., Xia, R., Kuang, H. \& Meyers, B. C. The diversification of plant NBS-LRR defense genes directs the evolution of microRNAs that target them. Mol. Biol. Evol. 33, 2692-2705 (2016).

23. Mun, J. H., Yu, H. J., Park, S. \& Park, B. S. Genome-wide identification of NBS-encoding resistance genes in Brassica rapa. Mol. Genet. Genom. 282, 617-631 (2009).

24. Jupe, F. et al. Identification and localisation of the NB-LRR gene family within the potato genome. BMC Genomics 13, 75 (2012).

25. Fischer, I., Diévart, A., Droc, G., Dufayard, J.-F. \& Chantret, N. Evolutionary dynamics of the leucine-rich repeat receptor-like kinase (LRR-RLK) subfamily in angiosperms. Plant Physiol. 170, 1595-1610 (2016).
26. Greeff, C., Roux, M., Mundy, J. \& Petersen, M. Receptor-like kinase complexes in plant innate immunity. Front. Plant Sci. 3, 1-7 (2012).

27. Fitzjohn, R. G. et al. How much of the world is woody? J. Ecol. 102, 1266-1272 (2014).

28. Gassmann, W., Hinsch, M. E. \& Staskawicz, B. J. The Arabidopsis RPS4 bacterial-resistance gene is a member of the TIR-NBS-LRR family of disease-resistance genes. Plant J. 20, 265-277 (1999).

29. Parker, J. E. et al. The Arabidopsis downy mildew resistance gene RPP5 shares similarity to the toll and interleukin-1 receptors with $\mathrm{N}$ and L6. Plant Cell 9, 879-894 (1997).

30. Enkhbayar, P., Kamiya, M., Osaki, M., Matsumoto, T. \& Matsushima, N. Structural principles of leucine-rich repeat (LRR) proteins. Proteins 54, 394-403 (2004).

31. Tobias, P. A. \& Guest, D. I. Tree immunity: growing old without antibodies Trends Plant Sci. 19, 367-370 (2014).

32. Jones, J. D. G. \& Dangl, J. L. The plant immune system. Nature 444 323-329 (2006).

33. Kremer, A. in Genome Mapping and Molecular Breeding in Plants: Forest Trees Vol. 7 (ed. Kole, C. R.) 165-187 (Springer-Verlag, Berlin, 2007).

34. Bodénès, $\mathrm{C}$. et al. Comparative mapping in the Fagaceae and beyond with EST-SSRs. BMC Plant Biol. 12, 153 (2012).

35. Bodénès, C., Chancerel, E., Ehrenmann, F., Kremer, A. \& Plomion, C. High-density linkage mapping and distribution of segregation distortion regions in the oak genome. DNA Res. 23, 115-124 (2016).

36. Faivre Rampant, P. et al. Analysis of BAC end sequences in oak, a keystone forest tree species, providing insight into the composition of its genome. BMC Genomics 12, 292 (2011).

37. Lesur, I. et al. A sample view of the pedunculate oak (Quercus robur) genome from the sequencing of hypomethylated and random genomic libraries. Tree Genet. Genomes 7, 1277-1285 (2011).

38. Saintagne, C. et al. Distribution of genomic regions differentiating oak species assessed by QTL detection. Heredity 92, 20-30 (2004).

39. Scotti-Saintagne, C. et al. Detection of quantitative trait loci controlling bud burst and height growth in Quercus robur L. Theor. Appl. Genet. 109 , 1648-1659 (2004)

40. Scotti-Saintagne, C., Bertocchi, E., Barreneche, T., Kremer, A. \& Plomion, C. Quantitative trait loci mapping for vegetative propagation in pedunculate oak. Ann. For. Sci. 62, 369-374 (2005).

41. Gailing, O. QTL analysis of leaf morphological characters in a Quercus robur full-sib family (Q. robur $\times$ Q. robur ssp. slavonica). Plant Biol. 10, 624-634 (2008).

42. Gailing, O., Langenfeld-Heyser, R., Polle, A. \& Finkeldey, R. Quantitative trait loci affecting stomatal density and growth in a Quercus robur progeny: implications for the adaptation to changing environments. Glob. Chang. Biol. 14, 1934-1946 (2008)

43. Casasoli, M. et al. Comparison of quantitative trait loci for adaptive traits between oak and chestnut based on an expressed sequence tag consensus map. Genetics 172, 533-546 (2006).

44. Parelle, J. et al. Quantitative trait loci of tolerance to waterlogging in a European oak (Quercus robur L.): physiological relevance and temporal effect patterns. Plant Cell Environ. 30, 422-434 (2007).

45. Brendel, O. et al. Quantitative trait loci controlling water use efficiency and related traits in Quercus robur L. Tree Genet. Genomes 4, 263-278 (2008).

46. Derory, J. et al. Contrasting relationships between the diversity of candidate genes and variation of bud burst in natural and segregating populations of European oaks. Heredity 104, 438-448 (2010).

47. Song, J. et al. X-ray computed tomography to decipher the genetic architecture of tree branching traits: oak as a case study. Tree Genet. Genomes 13, 5 (2017).

48. Rani, J., Chauhan, P. \& Tripathi, R. Li-Fi (Light Fidelity) - the future technology in wireless communication. Int. J. Appl. Eng. Res. 7, 1517-1520 (2012).

49. Zhang, H.-B. et al. Construction of BIBAC and BAC libraries from a variety of organisms for advanced genomics research. Nat. Protoc. 7, 479-499 (2012).

50. Plomion, C. et al. Decoding the oak genome: public release of sequence data, assembly, annotation and publication strategies. Mol. Ecol. Resour. 16, 254-265 (2016)

51. Marçais, G. \& Kingsford, C. A fast, lock-free approach for efficient parallel counting of occurrences of k-mers. Bioinformatics 27, 764-770 (2011).

52. Adams, M. D. et al. The genome sequence of Drosophila melanogaster. Science 287, 2185-2195 (2000)

53. Simpson, J. T. \& Durbin, R. Efficient construction of an assembly string graph using the FM-index. Bioinformatics 26, 367-373 (2010)

54. Boetzer, M. \& Pirovano, W. SSPACE-LongRead: scaffolding bacterial draft genomes using long read sequence information. BMC Bioinformatics 15, 211 (2014).

55. Joshi, N. \& Fass, J. Sickle: a sliding-window, adaptive, quality-based trimming tool for FastQ files (2011) 
56. Luo, R. et al. SOAPdenovo2: an empirically improved memory-efficient short-read de novo assembler. Gigascience 1, 18 (2012).

57. Korf, I. Gene finding in novel genomes. BMC Bioinformatics 5, 59 (2004)

58. Huang, S. et al. HaploMerger: reconstructing allelic relationships for polymorphic diploid genome assemblies. Genome Res. 22, 1581-1588 (2012).

59. Benson, G. Tandem Repeats Finder: a program to analyse DNA sequences. Nucleic Acids Res. 27, 573-578 (1999).

60. Smit, A. F. A., Hubley, R. \& Green, P. RepeatMasker Open-3.0 (1996).

61. Morgulis, A., Gertz, E. M., Schäffer, A. A. \& Agarwala, R. A fast and symmetric DUST implementation to mask low-complexity DNA sequences. J. Comput. Biol. 13, 1028-1040 (2006).

62. Price, A. L., Jones, N. C. \& Pevzner, P. A. De novo identification of repeat families in large genomes. Bioinformatics 21, 351-358 (2005).

63. Simão, F. A. et al. BUSCO: assessing genome assembly and annotation completeness with single-copy orthologs. Bioinformatics 19 3210-3212 (2015).

64. Flutre, T., Duprat, E., Feuillet, C. \& Quesneville, H. Considering transposable element diversification in de novo annotation approaches. PLOS ONE 6, el6526 (2011).

65. Hoede, C. et al. PASTEC: an automatic transposable element classification tool. PLoS ONE 9, e91929 (2014)

66. Quesneville, $\mathrm{H}$. et al. Combined evidence annotation of transposable elements in genome sequences. PLoS Comput. Biol. 1, 166-175 (2005).

67. Edgar, R. \& Myers, E. PILER: identification and classification of genomic repeats. Bioinformatics 21, i152-i158 (2005).

68. Bao, Z. \& Eddy, S. Automated de novo identification of repeat sequence families in sequenced genomes. Genome Res. 12, 1269-1276 (2002).

69. Huang, X. On global sequence alignment. Bioinformatics 10, 227-235 (1994).

70. Jurka, J. et al. Repbase Update, a database of eukaryotic repetitive elements. Cytogenet. Genome Res. 110, 462-467 (2005).

71. Finn, R. D. et al. Pfam: the protein families database. Nucleic Acids Res. 42, D222-D230 (2014).

72. Ahmed, I., Sarazin, A., Bowler, C., Colot, V. \& Quesneville, H. Genomewide evidence for local DNA methylation spreading from small RNAtargeted sequences in Arabidopsis. Nucleic Acids Res. 39, 6919-6931 (2011).

73. Foissac, S. et al. Genome annotation in plants and fungi: EuGene as a model platform. Curr. Bioinformatics 3, 87-97 (2008).

74. Schiex, T., Moisan, A. \& Rouzé, P. in Computational Biology. Lecture Notes in Computer Science Vol. 2066 (eds Gascuel, O. \& Sagot M. F.) 111-125 (Springer, Berlin, 2001).

75. Degroeve, S., Saeys, Y., De Baets, B., Rouzé, P. \& Van de Peer, Y. SpliceMachine: predicting splice sites from high-dimensional local context representations. Bioinformatics 21, 1332-1338 (2005).

76. Lesur, I. et al. The oak gene expression atlas: insights into Fagaceae genome evolution and the discovery of genes regulated during bud dormancy release. BMC Genomics 16, 112 (2015).

77. Quevillon, E. et al. InterProScan: protein domains identifier. Nucleic Acids Res. 33, W116-W120 (2005).

78. Krogh, A., Larsson, B., von Heijne, G. \& Sonnhammer, E. Predicting transmembrane protein topology with a hidden Markov model: application to complete genomes. J. Mol. Biol. 305, 567-580 (2001).

79. Kanehisa, M., Goto, S., Furumichi, M., Tanabe, M. \& Hirakawa, M. KEGG for representation and analysis of molecular networks involving diseases and drugs. Nucleic Acids Res. 38, D355-D360 (2010).

80. Marchler-Bauer, A. et al. CDD: specific functional annotation with the Conserved Domain Database. Nucleic Acids Res. 37, D205-D210 (2009).

81. Tatusov, R. L. et al. The COG database: an updated version includes eukaryotes. BMC Bioinformatics 4, 41 (2003).

82. Goodstein, D. M. et al. Phytozome: a comparative platform for green plant genomics. Nucleic Acids Res. 40, D1178-D1186 (2012).

83. Langmead, B. \& Salzberg, S. L. Fast gapped-read alignment with Bowtie 2. Nat. Methods 9, 357-359 (2012).

84. Li, H. et al. The Sequence Alignment/Map format and SAMtools. Bioinformatics 25, 2078-2079 (2009).

85. Guichoux, E., Lagache, L., Wagner, S., Léger, P. \& Petit, R. J. Two highly validated multiplexes (12-plex and 8-plex) for species delimitation and parentage analysis in oaks (Quercus spp.). Mol. Ecol. Resour. 11, 578-585 (2011).

86. Wang, J. Coancestry: a program for simulating, estimating and analysing relatedness and inbreeding coefficients. Mol. Ecol. Resour. 11, 141-145 (2011).

87. Lagache, L., Leger, J. B., Daudin, J. J., Petit, R. J. \& Vacher, C. Putting the biological species concept to the test: using mating networks to delimit species. PLoS ONE 8, 1-11 (2013).

88. Falush, D., Stephens, M. \& Pritchard, J. K. Inference of population structure using multilocus genotype data: dominant markers and null alleles. Mol. Ecol. Notes 7, 574-578 (2007).
89. Futschik, A. \& Schlötterer, C. The next generation of molecular markers from massively parallel sequencing of pooled DNA samples. Genetics $\mathbf{1 8 6}$, 207-218 (2010).

90. Kofler, R., Pandey, R. V. \& Schlötterer, C. PoPoolation2: identifying differentiation between populations using sequencing of pooled DNA samples (Pool-Seq). Bioinformatics 27, 3435-3436 (2011).

91. Kofler, R. et al. PoPoolation: a toolbox for population genetic analysis of next generation sequencing data from pooled individuals. PLOS ONE 6, e15925 (2011).

92. Li, H. Aligning sequence reads, clone sequences and assembly contigs with BWA-MEM. Preprint at https://arxiv.org/abs/1303.3997 (2013).

93. Salse, J., Abrouk, M., Murat, F., Quraishi, U. M. \& Feuillet, C. Improved criteria and comparative genomics tool provide new insights into grass paleogenomics. Brief. Bioinform. 10, 619-630 (2009).

94. Yang, Z. PAML 4: phylogenetic analysis by maximum likelihood. Mol. Biol. Evol. 24, 1586-1591 (2007).

95. Li, L., Stoeckert, C. J. \& Roos, D. S. OrthoMCL: identification of ortholog groups for eukaryotic genomes. Genome Res. 13 2178-2189 (2003)

96. Zdobnov, E. M. \& Apweiler, R. InterProScan - an integration platform for the signature-recognition methods in InterPro. Bioinformatics 17, 847-848 (2001).

97. De Bie, T., Cristianini, N., Demuth, J. P. \& Hahn, M. W. CAFE: a computational tool for the study of gene family evolution. Bioinformatics 22, 1269-1271 (2006).

98. Han, M. V., Thomas, G. W. C., Lugo-Martinez, J. \& Hahn, M. W. Estimating gene gain and loss rates in the presence of error in genome assembly and annotation using CAFE 3. Mol. Biol. Evol. 30 1987-1997 (2013)

99. Edgar, R. C. MUSCLE: a multiple sequence alignment method with reduced time and space complexity. BMC Bioinformatics 5, 113 (2004)

100. Borowiec, M. L. AMAS: a fast tool for alignment manipulation and computing of summary statistics. PeerJ 4, el660 (2016).

101. Hochberg, Y. \& Benjamini, Y. More powerful procedures for multiple statistical significance testing. Stat. Med. 9, 811-818 (1990).

102. Sasaki, T., Massaki, N. \& Kubo, T. Wolbachia variant that induces two distinct reproductive phenotypes in different hosts. Heredity 95 389-393 (2005).

103. Alexa, A., Rahnenführer, J. \& Lengauer, T. Improved scoring of functional groups from gene expression data by decorrelating GO graph structure. Bioinformatics 22, 1600-1607 (2006).

104. Lee, E. et al. Web Apollo: a web-based genomic annotation editing platform. Genome Biol. 14, R93 (2013).

105. Kalderimis, A. et al. InterMine: extensive web services for modern biology. Nucleic Acids Res. 42, W468-W472 (2014).

\section{Acknowledgements}

The main sources of funding were the ANR (GENOAK 2022-BSV6-009-02), INRA and CEA. T.L. was supported by fellowships from the ANR and a European Research Council Advanced Grant (ERC TREEPEACE FP7-339728). I. Lesur and T.A. were supported by fellowships from the ANR. N.F. received funding from the ANR (ARBRE ANR22-LABX-0002-02) and the ERC. J.C. was supported by the Swedish Foundation for Strategic Research.

\section{Author Contributions}

C.P., J.-M.A. and J.A. were the lead investigators and are joint first authors. T.L. and F.Murat are joint authors and contributed equally to the work. C.P. conceived and coordinated the project, supervised the research, and organized the main text of the manuscript and the supplementary materials, with contributions from all authors. T.L. estimated heterozygosity from the reference genome sequence and genetic diversity from pool-seq data, and performed somatic mutation analyses together with E.C. G.L.P. participated in the annotation jamboree, coordinated tissue sampling from the reference genotype and organized the collection of the genotypes used for genetic diversity and somatic mutation analyses. C.Bodénès established the composite linkage map of oak and participated in the annotation jamboree. I. Lesur identified the allelic gene pairs and tandem duplicated genes. I. Lesur also prepared the RNA-sequencing tracks for JBrowse, concatenated the functional annotations for the orthogroups and participated in the annotation jamboree. F.E. was involved in database development and prepared the quantitative trait loci tracks of JBrowse. E.G., C.L. and F.S. were involved in genomic DNA and RNA extraction, genotyping before the pooling of pedunculate oak genotypes and the genotyping of somatic mutations in the offspring. M.-L.D.-L. analysed the MLO gene family. A. Kremer contributed to the writing and critical review of the manuscript. B.B. provided critical comments on earlier versions of the manuscript, helped to reorganize the final version and reviewed the supplementary materials. J.B. performed the GO term enrichment and gene family expansion analysis comparing trees and herbaceous species. J.-M.A. directed the sequencing and assembly parts of the project. S.F., A.C., C.D.S., C.D., M.-A.M. and J.M. were involved in the bioinformatic analyses (BAC, genome and transcriptome). K.L., V.B., C. Besler, A.L. 
and S.M. prepared the libraries and undertook most of the sequencing activities. P.W. supervised the sequencing activity. J.A. led the genome annotation and TE analysis, organized the gene annotation jamboree, and directed the setting up of genome browsers and OakMine interfaces. N.F. performed the genome annotation and set up the web interfaces. I. Luyten and T.A. participated in the TE annotation. F. Maumus annotated the endovirus. C. Michotey was responsible for the insertion of genetic data into the GnpIS database. T.A. helped improve the REPET pipeline that was used to annotate the TEs and participated in the TE annotation. H.Q. supervised the TE analysis. F. Maurat and J.S. performed the macroevolutionary analyses. S.D., C. Marchal analysed the NBLRR gene family. A. Kohler and F. Martin annotated genes involved in biotic interactions with ectomycorrhizal fungi and jointly organized the gene annotation jamboree. I.H. D.C. and M.-B.B.-T. analysed the aquaporin gene family. A.H. and N.R. analysed the thioredoxin, glutaredoxin and glutathione transferase gene families. P.H., C.R. and A.V. were involved in the manual curation of candidate genes for gallotannin production. J.-C.L. annotated the laccase gene family. P.F.-R. annotated the BAC clone sequences. C.G., C.K. and O.R. analysed non-coding RNAs and carried out OrthoMCL analysis. H.B. provided HMW DNA for sequencing. M.S. and J.G.-P. analysed the MYB gene family. N.C. and A.D. analysed the LRR-RLK and LRR-RLP gene families. M.-L.B, S.H and M.T. analysed the SWEET gene family and expression levels, and annotated genes involved in ectomycorrhizal interactions. A.E.Z. carried out the prospective analysis to identify genes related to the tree habit and provided critical comments on earlier versions of the manuscript. J.C. and M.L. estimated the efficacy of purifying selection in oak. O.P., E.L. and N.P. were involved in the analysis of TEs and genome dynamics.

\section{Competing interests}

The authors declare no competing interests.

\section{Additional information}

Supplementary information is available for this paper at https://doi.org/10.1038/ s41477-018-0172-3.

Reprints and permissions information is available at www.nature.com/reprints. Correspondence and requests for materials should be addressed to C.P.

Publisher's note: Springer Nature remains neutral with regard to jurisdictional claims in published maps and institutional affiliations.

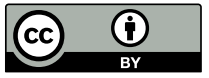

Open Access This article is licensed under a Creative Commons Attribution 4.0 International License, which permits use, sharing, adaptation, distribution and reproduction in any medium or format, as long as you give appropriate credit to the original author(s) and the source, provide a link to the Creative Commons license, and indicate if changes were made. The images or other third party material in this article are included in the article's Creative Commons license, unless indicated otherwise in a credit line to the material. If material is not included in the article's Creative Commons license and your intended use is not permitted by statutory regulation or exceeds the permitted use, you will need to obtain permission directly from the copyright holder. To view a copy of this license, visit http://creativecommons.org/licenses/by/4.0/. 


\section{Reporting Summary}

Nature Research wishes to improve the reproducibility of the work that we publish. This form provides structure for consistency and transparency in reporting. For further information on Nature Research policies, see Authors \& Referees and the Editorial Policy Checklist.

\section{Statistical parameters}

When statistical analyses are reported, confirm that the following items are present in the relevant location (e.g. figure legend, table legend, main text, or Methods section).

$\mathrm{n} / \mathrm{a} \mid$ Confirmed

$\bigotimes$ The exact sample size $(n)$ for each experimental group/condition, given as a discrete number and unit of measurement

$\square$ An indication of whether measurements were taken from distinct samples or whether the same sample was measured repeatedly

$\triangle$ The statistical test(s) used AND whether they are one- or two-sided

Only common tests should be described solely by name; describe more complex techniques in the Methods section.

Х $\square$ A description of all covariates tested

$\square$ A description of any assumptions or corrections, such as tests of normality and adjustment for multiple comparisons

$\square$ A full description of the statistics including central tendency (e.g. means) or other basic estimates (e.g. regression coefficient) AND

variation (e.g. standard deviation) or associated estimates of uncertainty (e.g. confidence intervals)

. For null hypothesis testing, the test statistic (e.g. $F, t, r$ ) with confidence intervals, effect sizes, degrees of freedom and $P$ value noted

Give $P$ values as exact values whenever suitable.

Х $\square$ For Bayesian analysis, information on the choice of priors and Markov chain Monte Carlo settings

Х $\square$ For hierarchical and complex designs, identification of the appropriate level for tests and full reporting of outcomes

Х $\square$ Estimates of effect sizes (e.g. Cohen's $d$, Pearson's $r$ ), indicating how they were calculated

$\varnothing$ Clearly defined error bars

State explicitly what error bars represent (e.g. SD, SE, CI)

Our web collection on statistics for biologists may be useful.

\section{Software and code}

Policy information about availability of computer code

Data collection

1/ sequencing data collection: we used Roche/454 (non operating anymore) and Illumina sequencing machines that included their own computer packages for DNA sequence collection. 2/ genotyping data collection: Allele calling from the MassArray iPLEX assay was processed in Typer Viewer v 4.0.26.75 software (Agena Bioscience).

Data analysis
custom-made scripts were made available through public web sites. This is clearly stated in the Ms.
We relied on $\mathrm{R}$ for statistical analyses.
The following public or commercial software were used: AMAS / Augustus / BLAST / Bowtie2 / bcftools / BWA-MEM / BLAT / bedtools / BioNJ / Celera assembler / COANCESTRY / CAFE version 3.1 / Censor / CLC genomics Workbench / Clustal-Omega / Cabog / ClustalW / DUST / EuGene / EggLib / FGENESH / FigTree V1.4.3 / Fastree 2.1.8 / FEELnc version 26.05.2015 / FeatureCounts / Ggplot2 / G-block v0.91b / Geneious 6.1.8 / HAPLOMERGE V1 / HMMER 3.0 / Interproscan v5.13-52.0 / infernal 1.1 / Jellyfish / LPmerge / LTRharvest / MAP / MuTect / MUSCLE / MUMmer / MAFFT version 5 / MEGA5 / MEGA6 / Newbler assembler (version MapAsmResearch-04/19/2010- patch-08/17/2010) / netGen2 / NUCmer / NBLR parser / OLC assembler / OrthoMCL / Picard / Popoolation2 / Popoolation / PAML 4/ PhyML 3.0 / Prank / RepeatMasker open-3.0/ RepeatScout / REPET / RAXML 7.7.2 / RNAmmer / SSPACE / Sickle / SNPA gene finder / SpliceMachine / Signl P /SAMtools / STRUCTURE / SOAPdenovo2 / SiLiX / S-MART / Seaview version 4 / Stringtie v1.0.1 / TRF / TargetP / TMHMM / Typer Viewer v4.0.26.75 / topGO 2.22.0 / tandem repeatsFinder / trimAl gt 0.2 / STAR 2.4.0i / tRNAscan-SE / taxize / TreeeDyn v198.3 / YASS

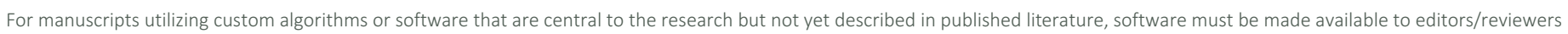

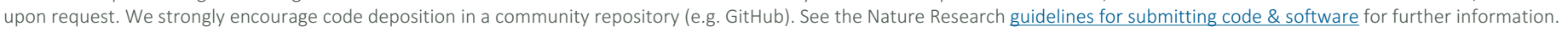


Policy information about availability of data

All manuscripts must include a data availability statement. This statement should provide the following information, where applicable:

- Accession codes, unique identifiers, or web links for publicly available datasets

- A list of figures that have associated raw data

- A description of any restrictions on data availability

The oak haploid genome assembly and corresponding annotation have been deposited in the European Nucleotide Archive under project accession code PRJEB19898. Other sequence release data are indicated in Supplementary tables 1, 13, 14 and 19 and Supplementary Data Set 10. We also invite readers to download data stored at the URLs indicated in section 6 (Web resources) as well as in the oakgenome web site: http://www.oakgenome.fr.

\section{Field-specific reporting}

Please select the best fit for your research. If you are not sure, read the appropriate sections before making your selection.

\ Life sciences

Behavioural \& social sciences

For a reference copy of the document with all sections, see nature.com/authors/policies/ReportingSummary-flat.pdf

\section{Life sciences}

\section{Study design}

All studies must disclose on these points even when the disclosure is negative.

Sample size selection of tree and herbaceous genomes for comparison of gene content were made according to the quality of publicaly available genom assemblies: see Online Methods section "Detection of significant expansion/contraction in woody perennials. "

Data exclusions As explained in the Method section (line 711-713) we excluded from our initial population screening : related genotypes (3) as well as introgressed genotypes (8), leaving as much as possible unrelated and not introgressed genotypes for population genetics analysis.

Replication

It is very important to stress that somatic mutations we deemed reliable were detected by comparing multiple sequencing libraries, taking into account the chronology of branch development. This is a much more powerful validation than would have been provided by the technical Sanger validation. In our approach, polymorphisms generated by assembly errors would be very unlikely i/ to show differences among the libraries for the different branches, and ii/ to follow a temporal pattern coherent with the chronology of branch development. Regarding Sanger validation, according to a recent study in humans (Beck et al. 2016, https://www.ncbi.nlm.nih.gov/pmc/articles/PMC4878677/), Sanger sequencing was more likely to incorrectly refute a true positive variant from NGS than to correctly identify a false positive. In addition, Sanger sequencing appears to have low sensitivity for low frequency variants, as expected for the vast majority of somatic mutations. We therefore preferred to rely on our comparative approach.

Randomization We compared estimates for genetic between genes from expanded, contracted, and unchanged gene families (orthogroups) in oak. We accounted for the different gene family sizes, by randomly sampling 1000 genes from each of these three categories and repeating the operation 100 times.

Blinding

genotype calls obtained from the Typer Viewersoftware of the mass array spectrometer were controlled by two people (according to a complete double-blind design), and considered as valid when the two calls were identicall.

\section{Materials \& experimental systems}

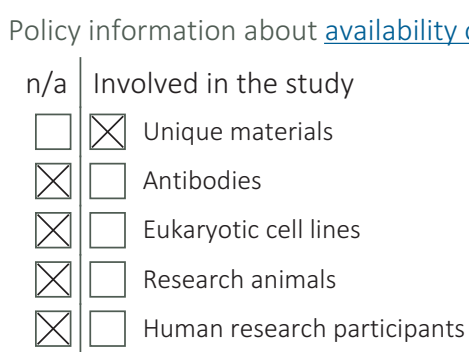

Unique materials

Obtaining unique materials

the genotype selected to sequence the oak genome is a 100 year-old tree in our experiemntal station. Still living and plant material are available upon request. 
Antibodies

Antibodies used

Describe all antibodies used in the study; as applicable, provide supplier name, catalog number, clone name, and lot number

Validation

Describe the validation of each primary antibody for the species and application, noting any validation statements on the manufacturer's website, relevant citations, antibody profiles in online databases, or data provided in the manuscript.

Eukaryotic cell lines

Policy information about cell lines

Cell line source(s)

State the source of each cell line used.

Authentication

Describe the authentication procedures for each cell line used OR declare that none of the cell lines used were authenticated.

Mycoplasma contamination

Confirm that all cell lines tested negative for mycoplasma contamination OR describe the results of the testing for mycoplasma contamination OR declare that the cell lines were not tested for mycoplasma contamination.

Commonly misidentified lines (See ICLAC register)

Name any commonly misidentified cell lines used in the study and provide a rationale for their use.

Research animals

Policy information about studies involving animals; ARRIVE guidelines recommended for reporting animal research

Animals/animal-derived materials

For laboratory animals, report species, strain, sex and age OR for animals observed in or captured from the field, report species, sex and age where possible.

Human research participants

Policy information about studies involving human research participants

Population characteristics Describe the covariate-relevant population characteristics of the human research participants (e.g. age, gender, genotypic information, past and current diagnosis and treatment categories).

\section{Method-specific reporting}

$\mathrm{n} / \mathrm{a}$ Involved in the study

\ $\square$ chip-seq

$\triangle \square$ Flow cytometry

Х Magnetic resonance imaging

ChIP-seq

Data deposition

Х Confirm that both raw and final processed data have been deposited in a public database such as GEO.

$\square$ Confirm that you have deposited or provided access to graph files (e.g. BED files) for the called peaks.

Data access links

May remain private before publication.

Files in database submission

Genome browser session (e.g. UCSC)

Methodology

Replicates

Sequencing depth

Antibodies

Peak calling parameters

Data quality
For "Initial submission" or "Revised version" documents, provide reviewer access links. For your "Final submission" document, provide a link to the deposited data.

Provide a list of all files available in the database submission.

Provide a link to an anonymized genome browser session for "Initial submission" and "Revised version" documents only, to enable peer review. Write "no longer applicable" for "Final submission" documents.
Describe the experimental replicates, specifying number, type and replicate agreement.

Describe the sequencing depth for each experiment, providing the total number of reads, uniquely mapped reads, length of reads and whether they were paired-or single-end.

Describe the antibodies used for the ChIP-seq experiments; as applicable, provide supplier name, catalog number, clone name, and lot number.

Specify the command line program and parameters used for read mapping and peak calling, including the ChIP, control and index files used.

Describe the methods used to ensure data quality in full detail, including how many peaks are at FDR 5\% and above 5-fold 


\section{Flow Cytometry}

Plots

Confirm that:

$\square$ The axis labels state the marker and fluorochrome used (e.g. CD4-FITC).

$\square$ The axis scales are clearly visible. Include numbers along axes only for bottom left plot of group (a 'group' is an analysis of identical markers).

$\square$ All plots are contour plots with outliers or pseudocolor plots.

$\square$ A numerical value for number of cells or percentage (with statistics) is provided.

Methodology

Sample preparation

Instrument

Software

Cell population abundance

Gating strategy
Describe the sample preparation, detailing the biological source of the cells and any tissue processing steps used.

Identify the instrument used for data collection, specifying make and model number.

Describe the software used to collect and analyze the flow cytometry data. For custom code that has been deposited into a community repository, provide accession details.

Describe the abundance of the relevant cell populations within post-sort fractions, providing details on the purity of the samples and how it was determined.

Describe the gating strategy used for all relevant experiments, specifying the preliminary FSC/SSC gates of the starting cell population, indicating where boundaries between "positive" and "negative" staining cell populations are defined.

Tick this box to confirm that a figure exemplifying the gating strategy is provided in the Supplementary Information.

\section{Magnetic resonance imaging}

\section{Experimental design}

\section{Design type}

Design specifications

Behavioral performance measures

Acquisition

Imaging type(s)

Field strength

Sequence \& imaging parameters

Area of acquisition

Diffusion MRI

Used

Preprocessing

Preprocessing software

Normalization

Normalization template
Indicate task or resting state; event-related or block design.

Specify the number of blocks, trials or experimental units per session and/or subject, and specify the length of each trial or block (if trials are blocked) and interval between trials.

State number and/or type of variables recorded (e.g. correct button press, response time) and what statistics were used to establish that the subjects were performing the task as expected (e.g. mean, range, and/or standard deviation across subjects).

Specify: functional, structural, diffusion, perfusion.

Specify in Tesla

Specify the pulse sequence type (gradient echo, spin echo, etc.), imaging type (EPI, spiral, etc.), field of view, matrix size, slice thickness, orientation and TE/TR/flip angle.

State whether a whole brain scan was used OR define the area of acquisition, describing how the region was determined.

Not used

Provide detail on software version and revision number and on specific parameters (model/functions, brain extraction segmentation, smoothing kernel size, etc.).

If data were normalized/standardized, describe the approach(es): specify linear or non-linear and define image types used for transformation OR indicate that data were not normalized and explain rationale for lack of normalization.

Describe the template used for normalization/transformation, specifying subject space or group standardized space (e.g. original Talairach, MNI305, ICBM152) OR indicate that the data were not normalized. 
Noise and artifact removal

Volume censoring

Statistical modeling \& inference

Model type and settings

Effect(s) tested

Specify type of analysis:

Statistic type for inference (See Eklund et al. 2016)

Correction

Models \& analysis

$\mathrm{n} / \mathrm{a}$ Involved in the study

$\square \square$ Functional and/or effective connectivity

$\square$ Graph analysis

$\square \square$ Multivariate modeling or predictive analysis

Functional and/or effective connectivity

Graph analysis

Multivariate modeling and predictive analysis Carlo)
Describe your procedure(s) for artifact and structured noise removal, specifying motion parameters, tissue signals and physiological signals (heart rate, respiration).

Define your software and/or method and criteria for volume censoring, and state the extent of such censoring

Specify type (mass univariate, multivariate, RSA, predictive, etc.) and describe essential details of the model at the first and second levels (e.g. fixed, random or mixed effects; drift or auto-correlation).

Define precise effect in terms of the task or stimulus conditions instead of psychological concepts and indicate whether ANOVA or factorial designs were used.

\section{Whole brain $\square$ ROI-based $\square$ Both}

Specify voxel-wise or cluster-wise and report all relevant parameters for cluster-wise methods.

Describe the type of correction and how it is obtained for multiple comparisons (e.g. FWE, FDR, permutation or Monte

Report the measures of dependence used and the model details (e.g. Pearson correlation, partia correlation, mutual information).

Report the dependent variable and connectivity measure, specifying weighted graph or binarized graph, subject-or group-level, and the global and/or node summaries used (e.g. clustering coefficient, efficiency, etc.)

Specify independent variables, features extraction and dimension reduction, model, training and evaluation metrics.

\section{Behavioural \& social sciences}

\section{Study design}

All studies must disclose on these points even when the disclosure is negative.

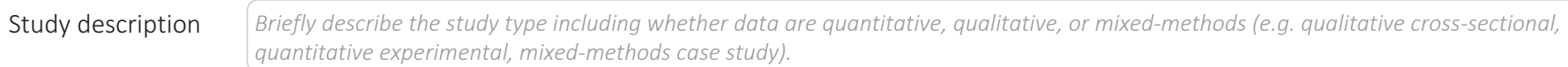

Research sample State the research sample (e.g. Harvard university undergraduates, villagers in rural India) and provide relevant demographic information (e.g. age, sex) and indicate whether the sample is representative. Provide a rationale for the study sample chosen. For studies involving existing datasets, please describe the dataset and source.

Sampling strategy Describe the sampling procedure (e.g. random, snowball, stratified, convenience). Describe the statistical methods that were used to predetermine sample size OR if no sample-size calculation was performed, describe how sample sizes were chosen and provide a rationale for why these sample sizes are sufficient. For qualitative data, please indicate whether data saturation was considered, and what criteria were used to decide that no further sampling was needed.

Data collection

Provide details about the data collection procedure, including the instruments or devices used to record the data (e.g. pen and paper computer, eye tracker, video or audio equipment) whether anyone was present besides the participant(s) and the researcher, and whether the researcher was blind to experimental condition and/or the study hypothesis during data collection.

Timing

Indicate the start and stop dates of data collection. If there is a gap between collection periods, state the dates for each sample cohort.

Data exclusions

If no data were excluded from the analyses, state so OR if data were excluded, provide the exact number of exclusions and the rationale behind them, indicating whether exclusion criteria were pre-established.

Non-participation

State how many participants dropped out/declined participation and the reason(s) given OR provide response rate OR state that no participants dropped out/declined participation.

Randomization

If participants were not allocated into experimental groups, state so OR describe how participants were allocated to groups, and if 
